\title{
A Review on the Structural and Metabolic Biomarkers in Migraine
}

\section{Fereshteh Dalouchi ${ }^{1,2}$, Fatemeh Moradi $^{3}$, Sayed Mostafa Modarres Mousavi ${ }^{1,4}$, Fariba Karimzadeh ${ }^{\text {* }}$}

${ }^{1}$ Shefa Neuroscience Research Center, Khatam Alanbia Hospital, Tehran, Iran

${ }^{2}$ Department of Physiology, Iran University of Medical Sciences, Tehran, Iran

${ }^{3}$ Department of Anatomy, Faculty of Medicine, Iran University of Medical Sciences, Tehran, Iran

${ }^{4}$ Department of Nanobiotechnology, Faculty of Biological Sciences, Tarbiat Modares University, Tehran, Iran

${ }^{5}$ Cellular and Molecular Research Center, Iran University of Medical Sciences, Tehran, Iran

\section{Article Info:}

\section{A BSTRACT}

Introduction: Migraine is a neurovascular illness with repeated attacks of modest to intense headache, lasting 4-72 hours and linked to nausea, vomiting, and sensitivity to light and noise. Migraine is often associated with asthma, anxiety, depression, and chronic pain. Recent development in basic and clinical investigations has augmented our grasp of the pathophysiology of these headaches. New drugs and treatment for the improvement of these headaches are emergent. Therefore, biomarkers provide a dynamic and a potent indicator to understanding the spectrum of neurological disease with applications in observational and analytic epidemiology, randomized clinical trials, screening and analysis and forecast. Biomarkers can also reflect the entire spectrum of disease from the earliest manifestations to the terminal stages. Conclusion: This review discusses the definition and uses of migraine biomarkers, particularly their role in diagnosis, risk stratification and management of disease.

Key words:

1. Migraine Disorders

2. Biomarkers

3. Headache

4. Brain

*Corresponding Author: Fariba Karimzadeh

E-mail: kaimzade.f@iums.ac.ir 


\title{
مرورى بر نشانگرهاى زيستى ساختارى و متابوليتى در ميكرن
}

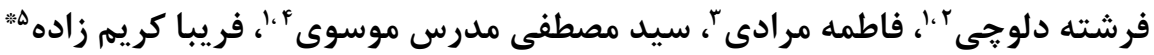

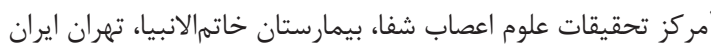

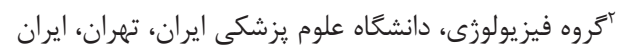

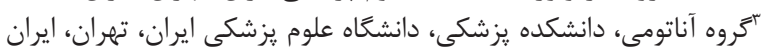

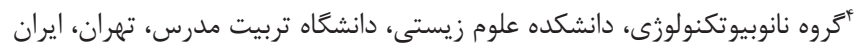

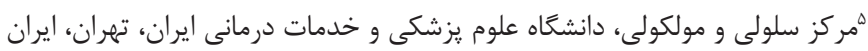

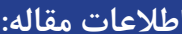

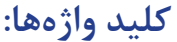

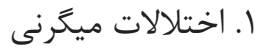
r.

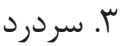
t

مقدمص: ميكرن يك بيمارى عصبى عروقى با حملات مكرر سردرد خفيف تا شديد است و به مدت

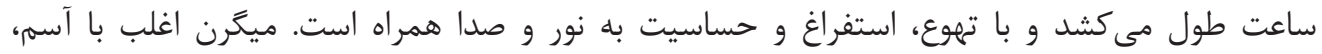

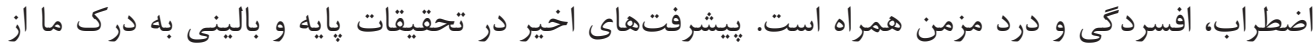

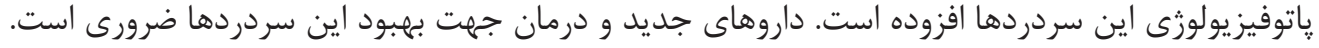

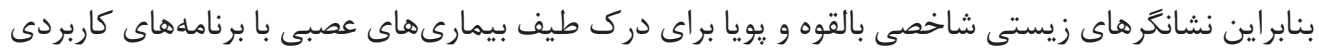

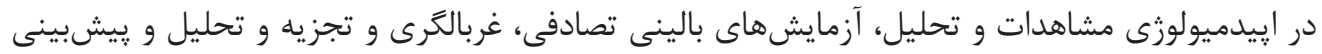

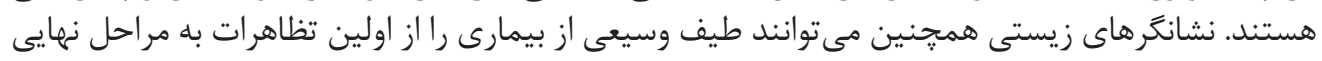

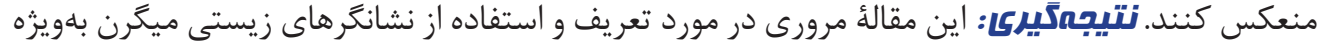

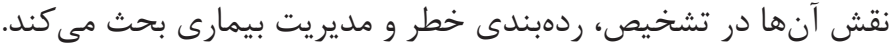

" نويسنده مسئول: فريبا كريم زاده آدرس الكترونيكى: kaimzade.f@iums.ac.ir 


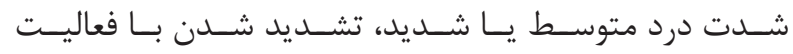

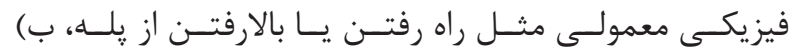

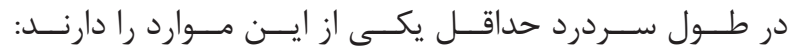

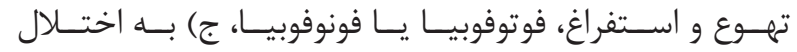

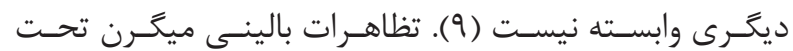

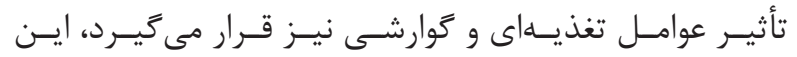

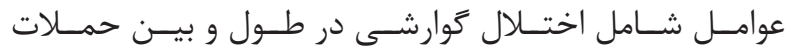

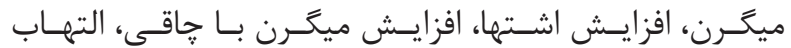

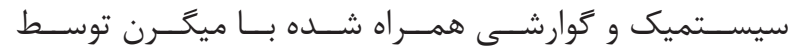

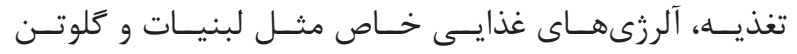

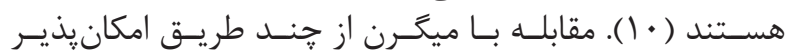

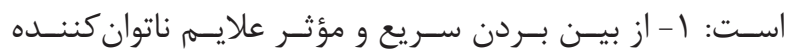

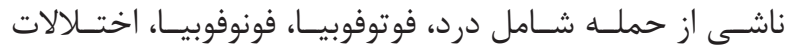

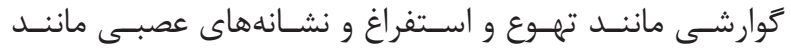

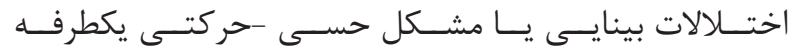

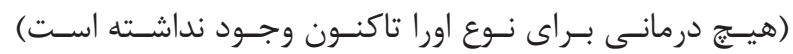

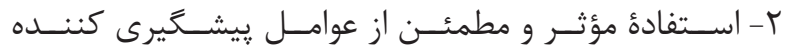

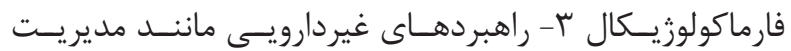

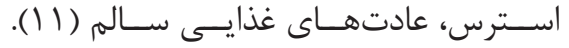

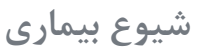

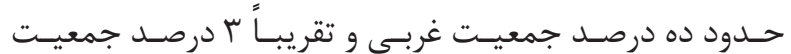

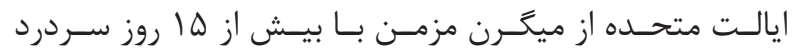

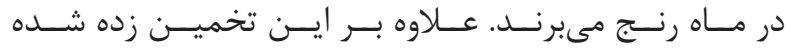

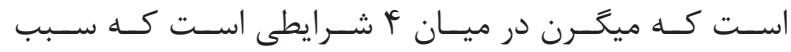

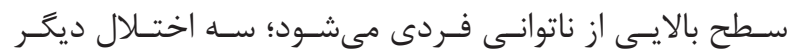

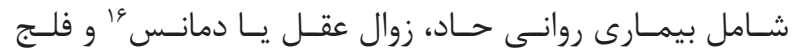

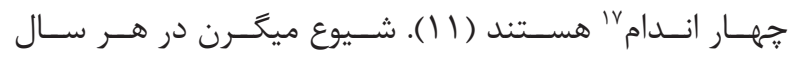

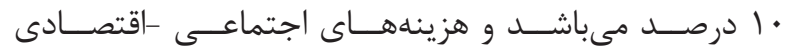

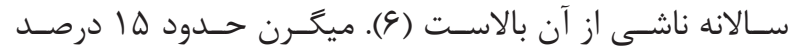

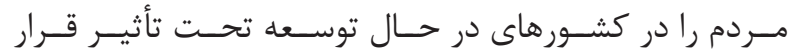

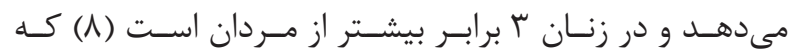

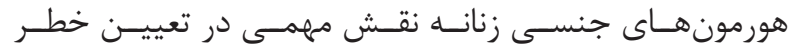

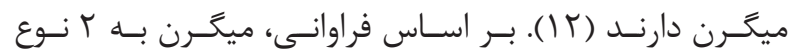

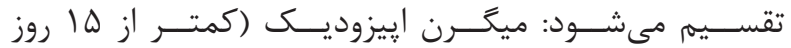

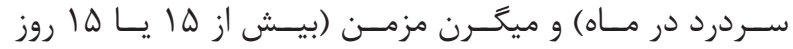

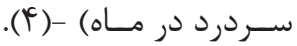

عوارض جانبى بيمارى

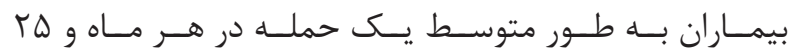

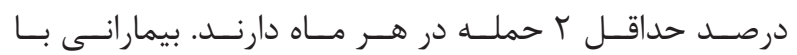

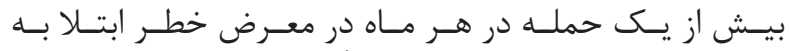

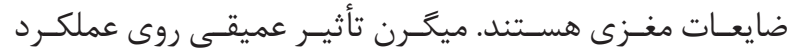

${ }^{1}$ Migraine with aura

${ }^{2}$ Migraine without aura

${ }^{3}$ New persistent daily headache

${ }^{4}$ Trigeminal autonomic cephalalgia

${ }^{5}$ Tension-type headache

${ }^{6}$ Photophobia

${ }^{7}$ Phonophobia

${ }^{8}$ Frontotemporal

${ }^{9}$ Ocular
مقدمه

تعريف بيمارى

جمعيـت زيـادى از جهـان از سـردرد رنـج مى برنسـ، بـهـ طـور

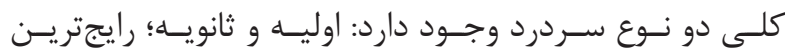

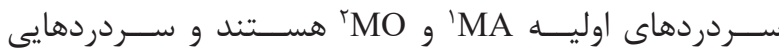
اوليـهاى كــه كمتــر رايــج هســتهند شــامل: هTH،

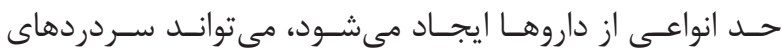

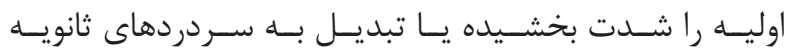

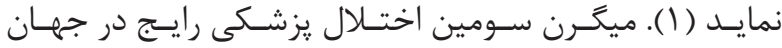

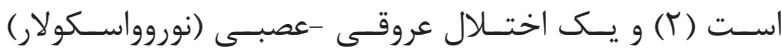

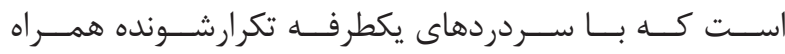

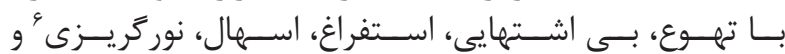

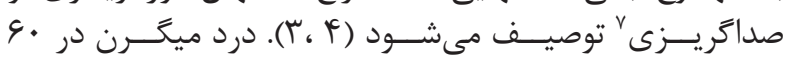

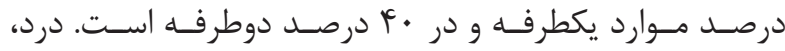

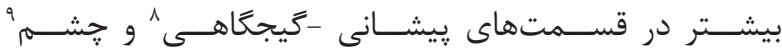

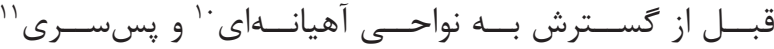

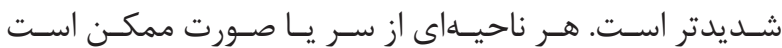

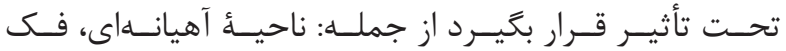

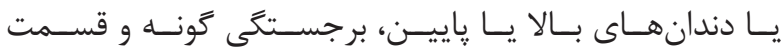

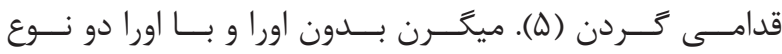

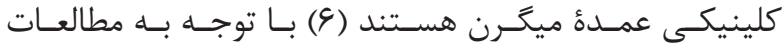

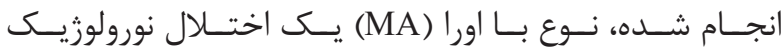

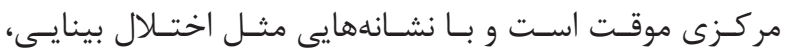

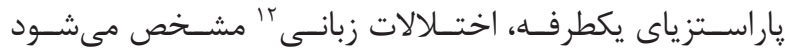

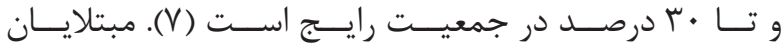

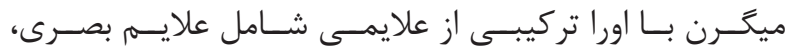

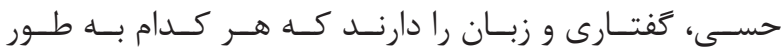

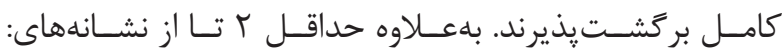

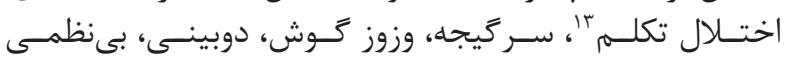

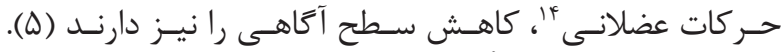

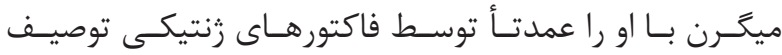

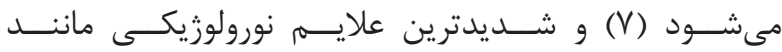

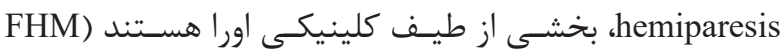

(A) $)^{10}$ (SHM L

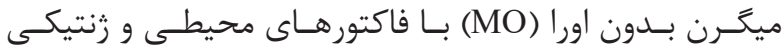

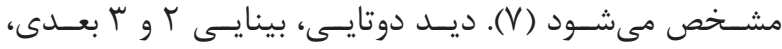

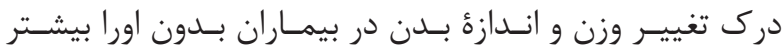

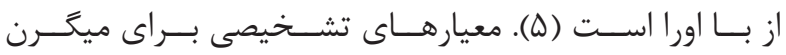

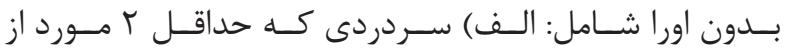

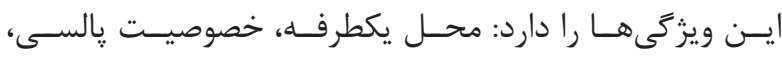

${ }^{10}$ Parietal

${ }^{11}$ Occipital

${ }^{12}$ Dysphasia

${ }^{13}$ Dysarthria

${ }^{14}$ Ataxia

${ }^{15}$ Familial or sporadic hemiplegic migraine

${ }^{16}$ Dementia

${ }^{17}$ Quadriplegia 


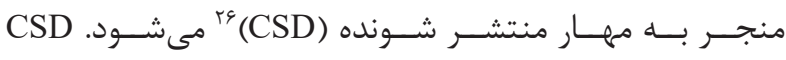

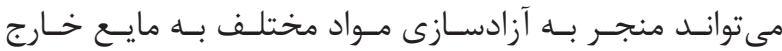

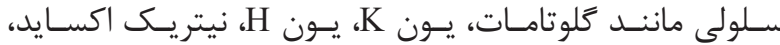

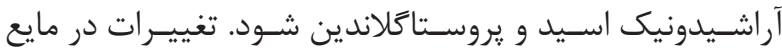

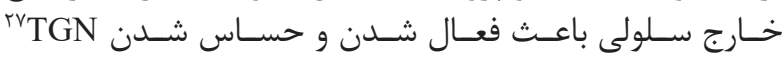

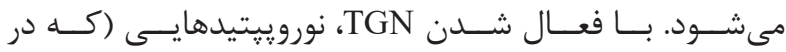

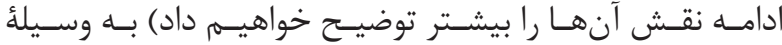

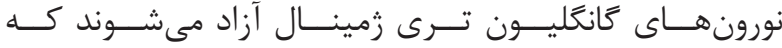

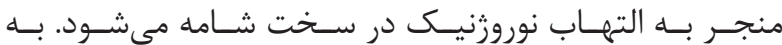

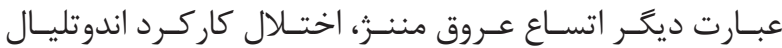

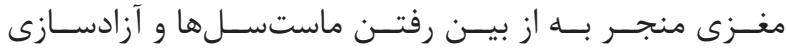

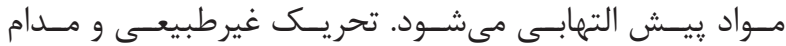

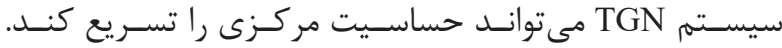

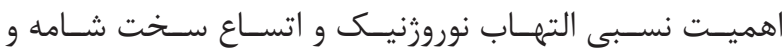

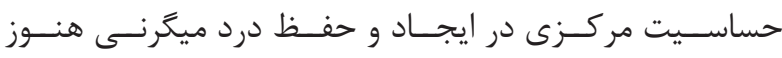

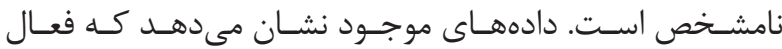

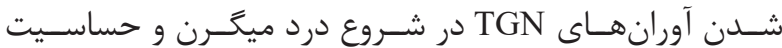

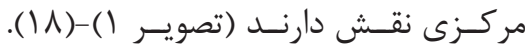

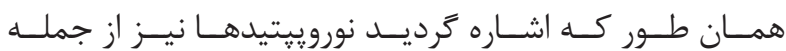

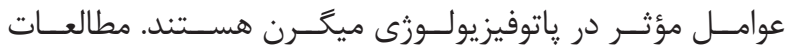

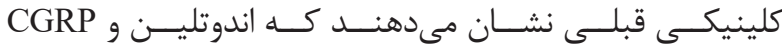

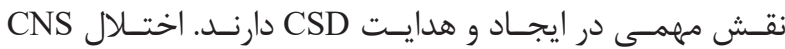

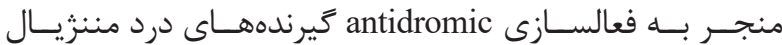

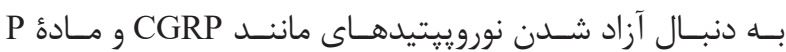

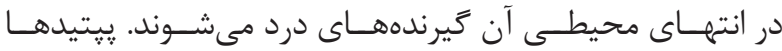

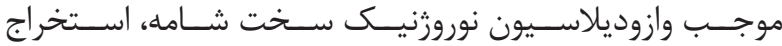

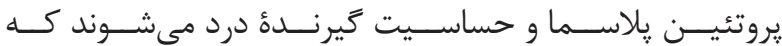

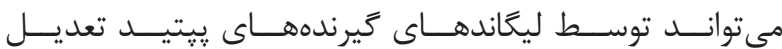

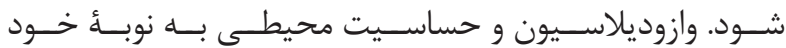

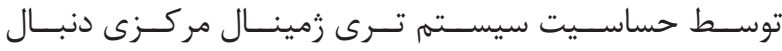

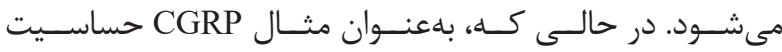

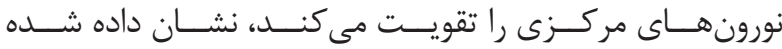

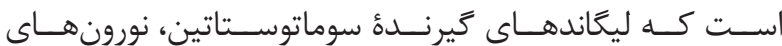

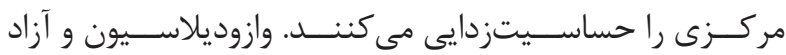

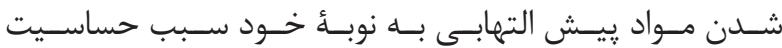

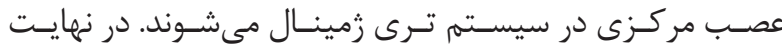

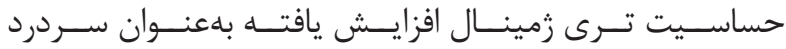

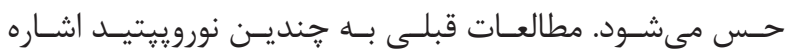

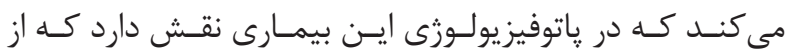

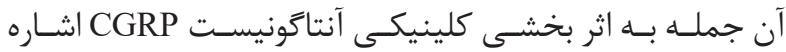

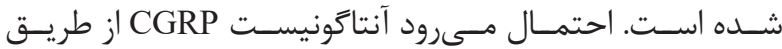

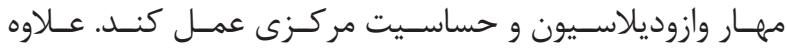

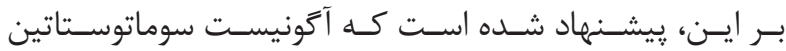

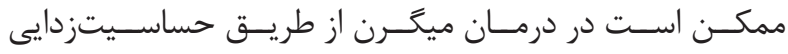

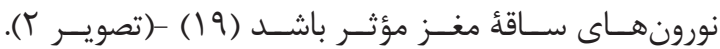

${ }^{18}$ Hypothalamic-pituitary-adernal

${ }^{19}$ Prefrontal cortex

${ }^{20}$ Endothelial NOS

${ }^{21}$ Neuronal NOS

${ }^{22}$ Inducible NOS

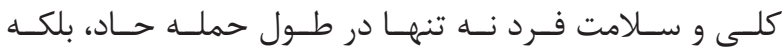

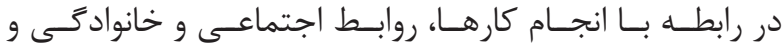

موفقيـت در امــور دارد (^).

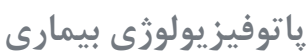

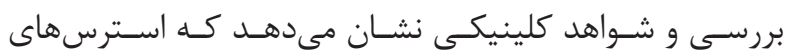

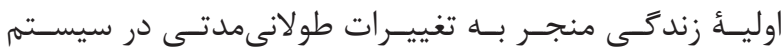

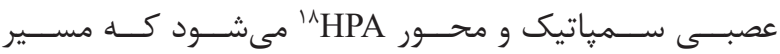

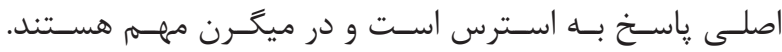

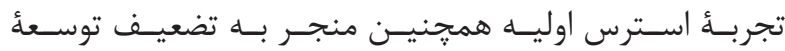

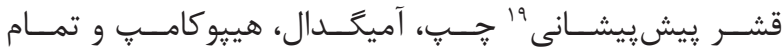

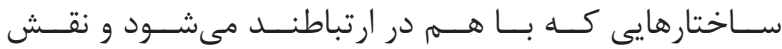

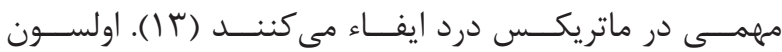

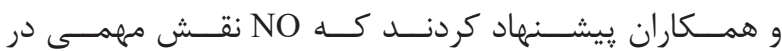

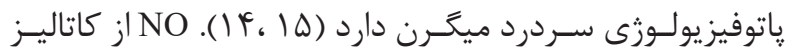

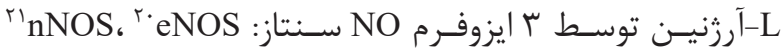

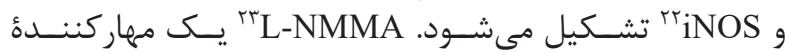

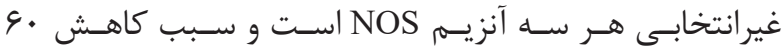

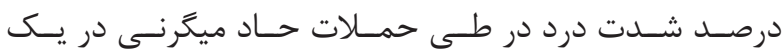

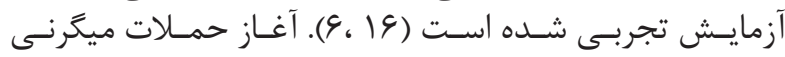

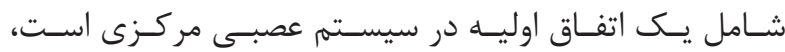

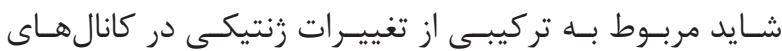

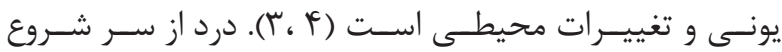

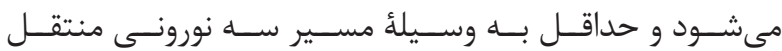

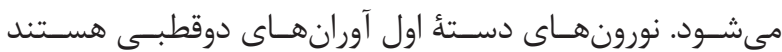

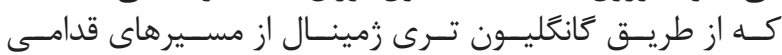

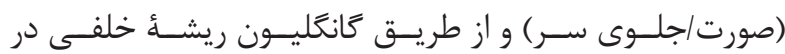

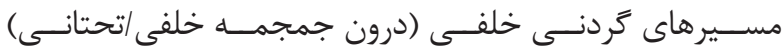

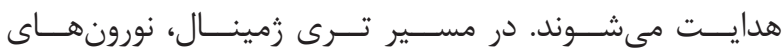

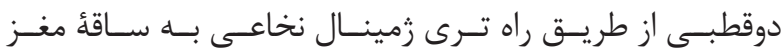

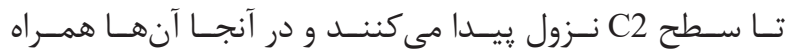

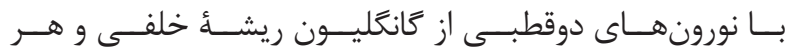

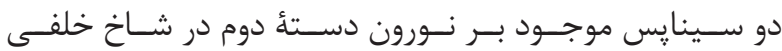

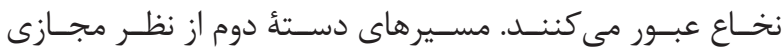

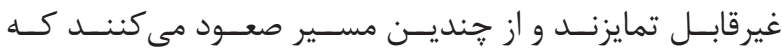

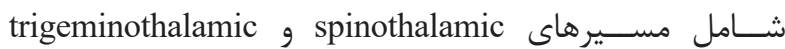

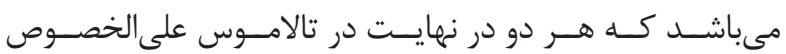
هسـته

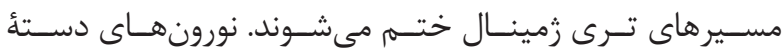

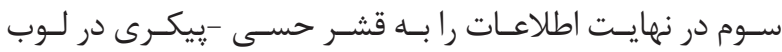

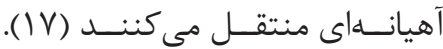

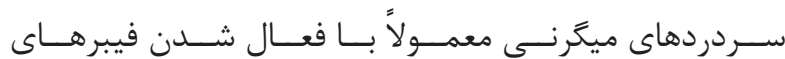

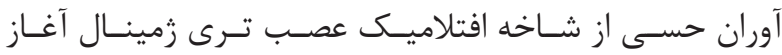

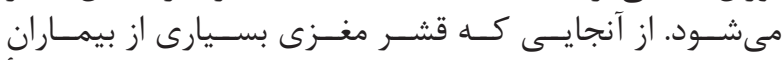

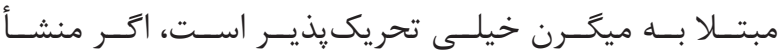

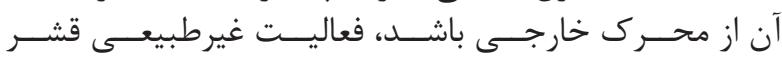

${ }^{23}$ NG-LMonomethyl arginine

${ }^{24}$ Ventral postero-lateral

${ }^{25}$ Ventral postero-medial

${ }^{26}$ Cortical spreading depression

${ }^{27}$ Trigeminal nerve 


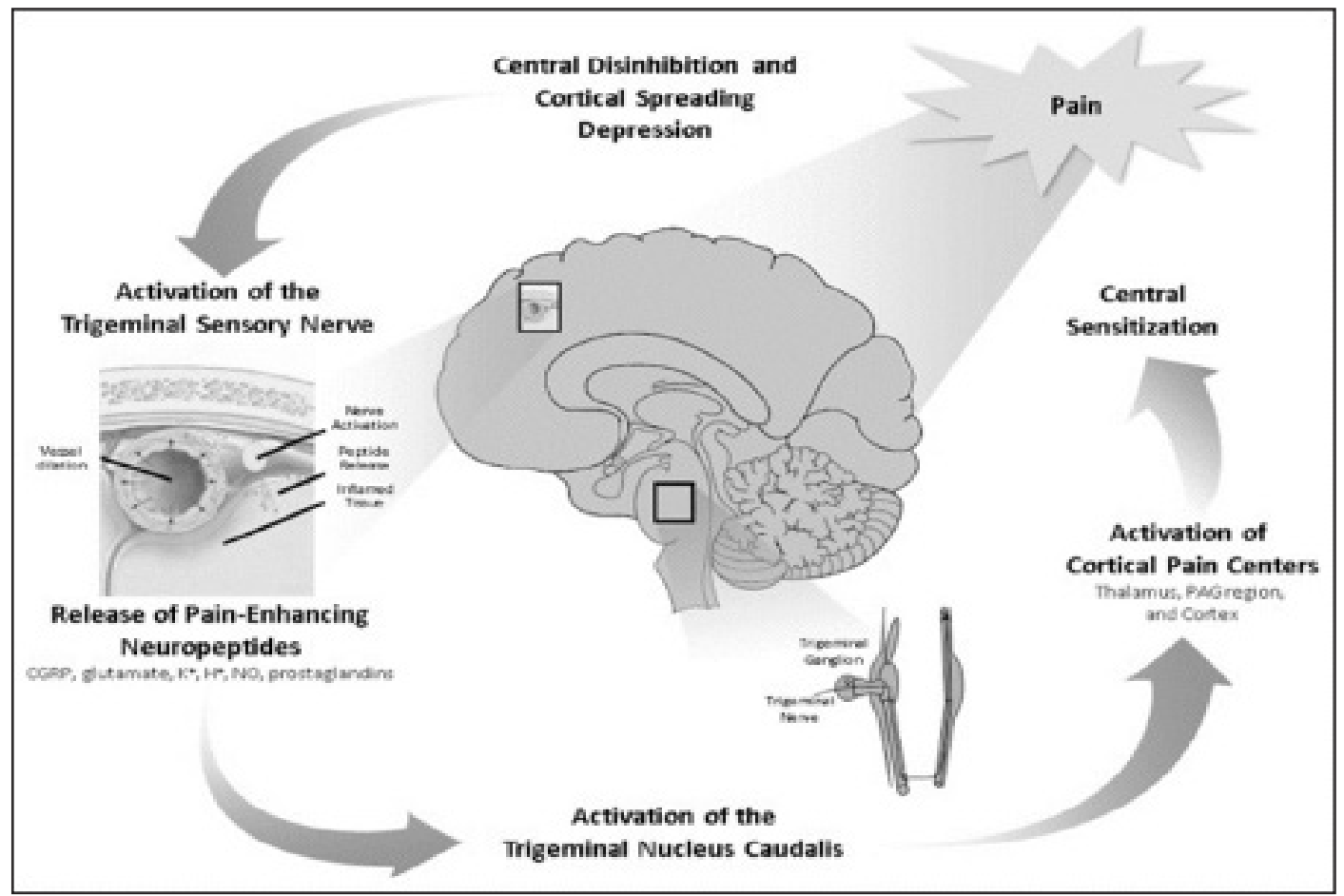

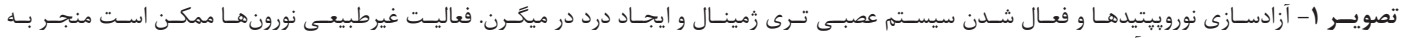

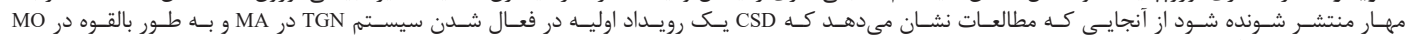

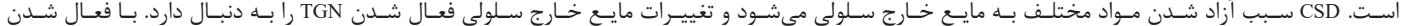

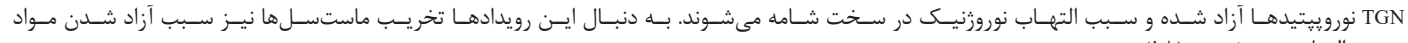
يَيـش التهابـى مى شـوند (1) (1).

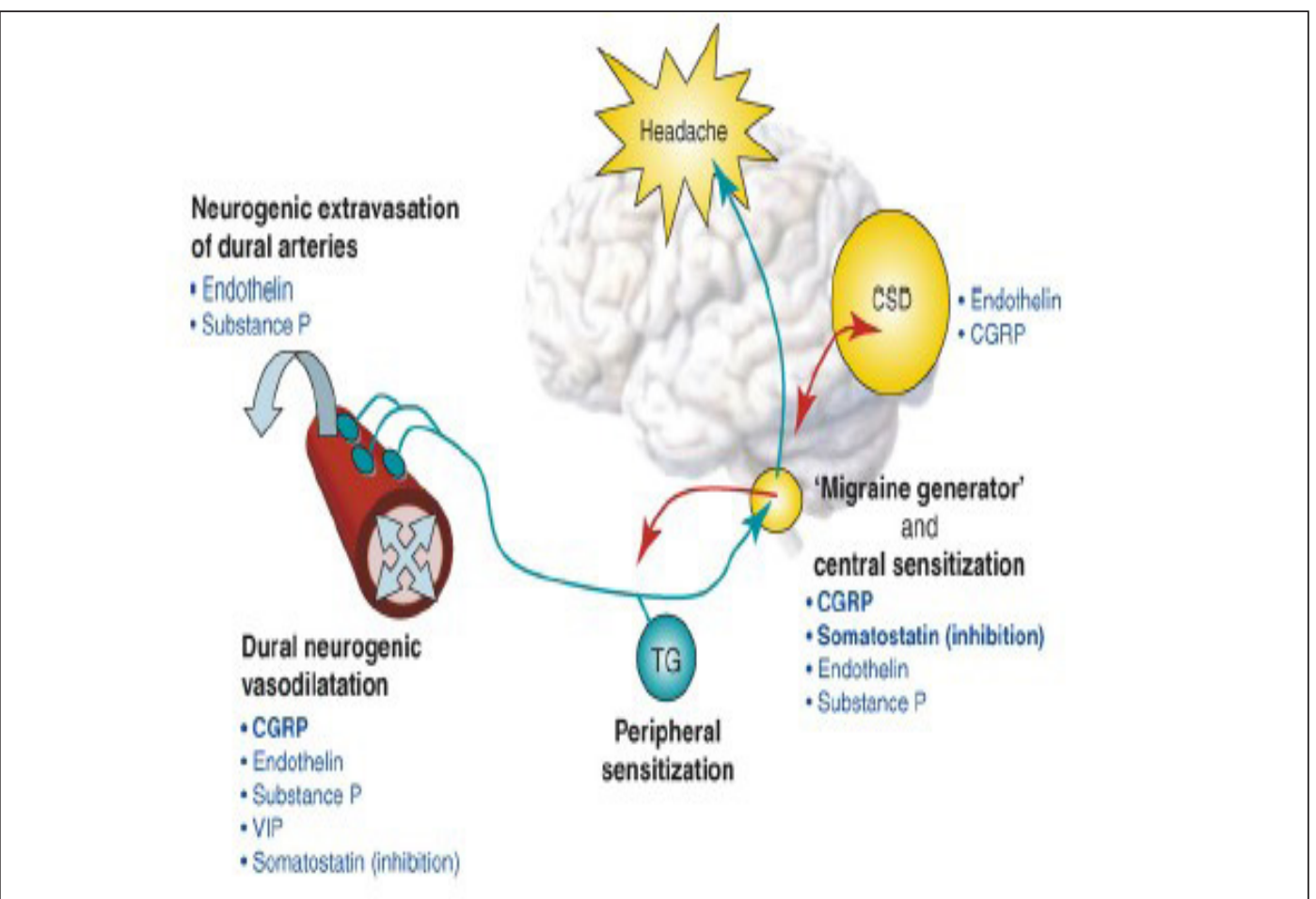

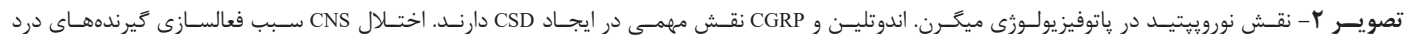

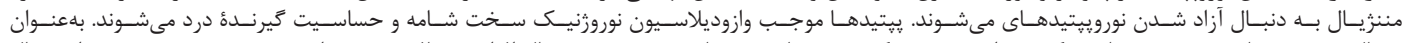

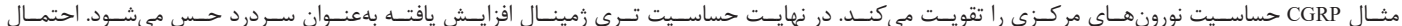

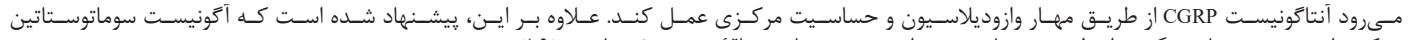

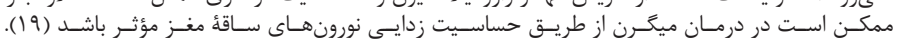




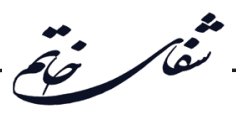

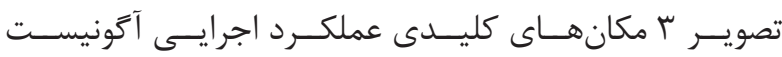

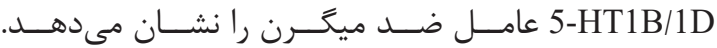

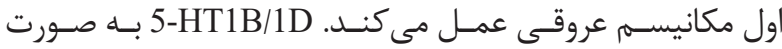

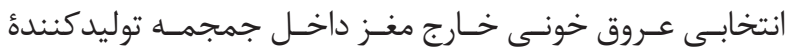

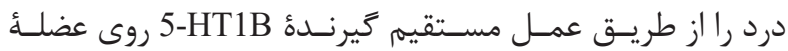

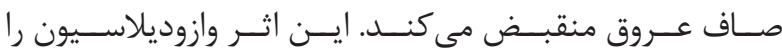

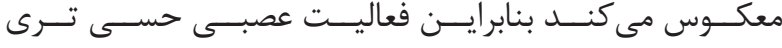

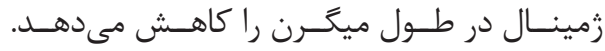

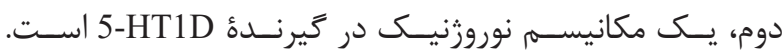

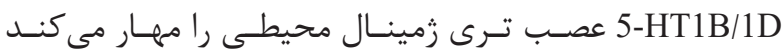

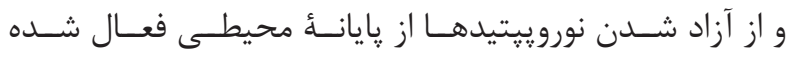

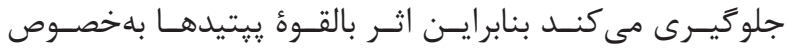
| ا حـذف مى كنــد.

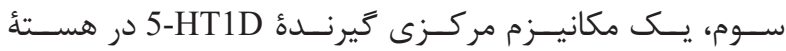

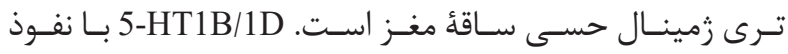

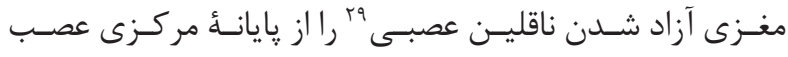

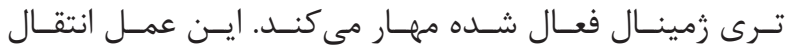

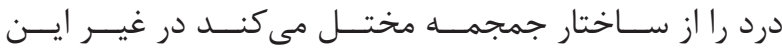

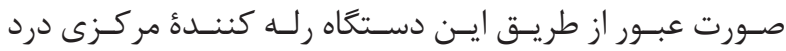

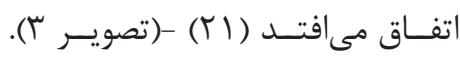

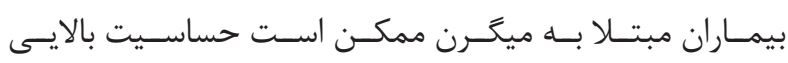

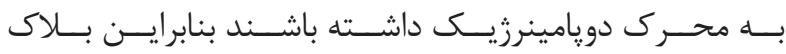

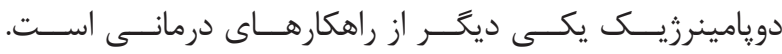

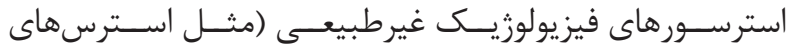

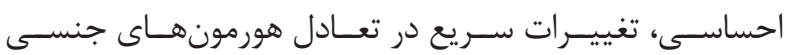

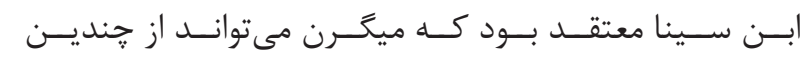

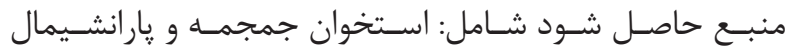

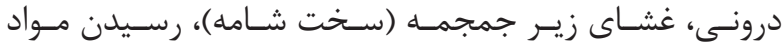

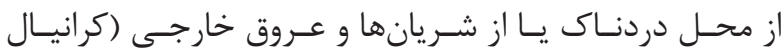

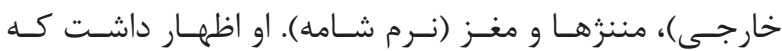

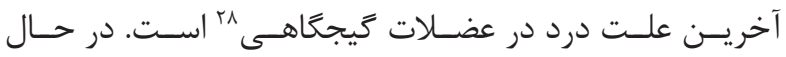

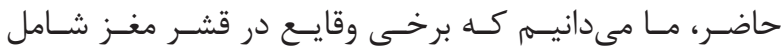

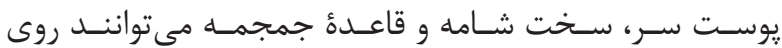

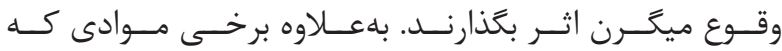

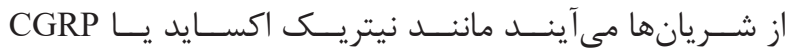

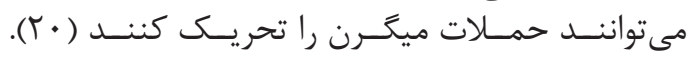
درمان بيمارى

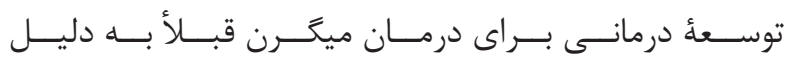

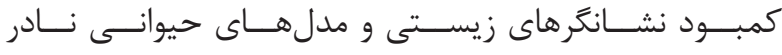

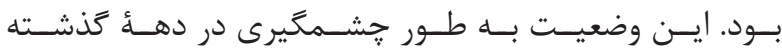

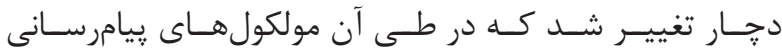

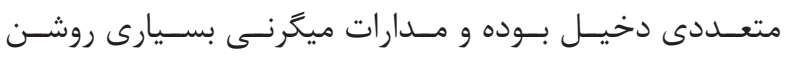

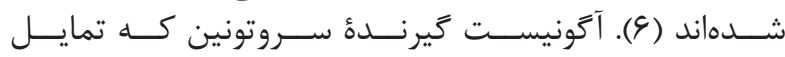

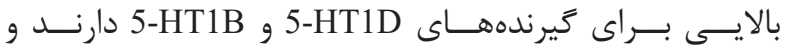

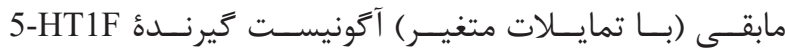

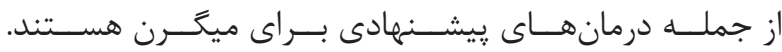

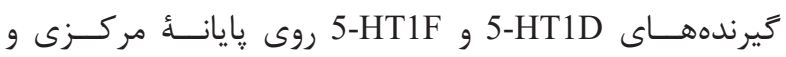

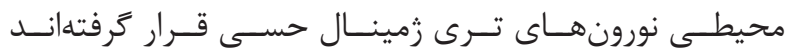

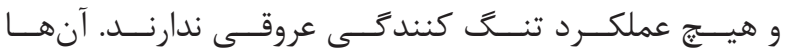

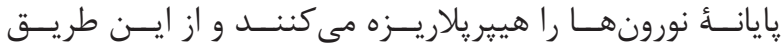

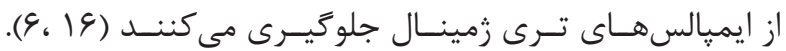

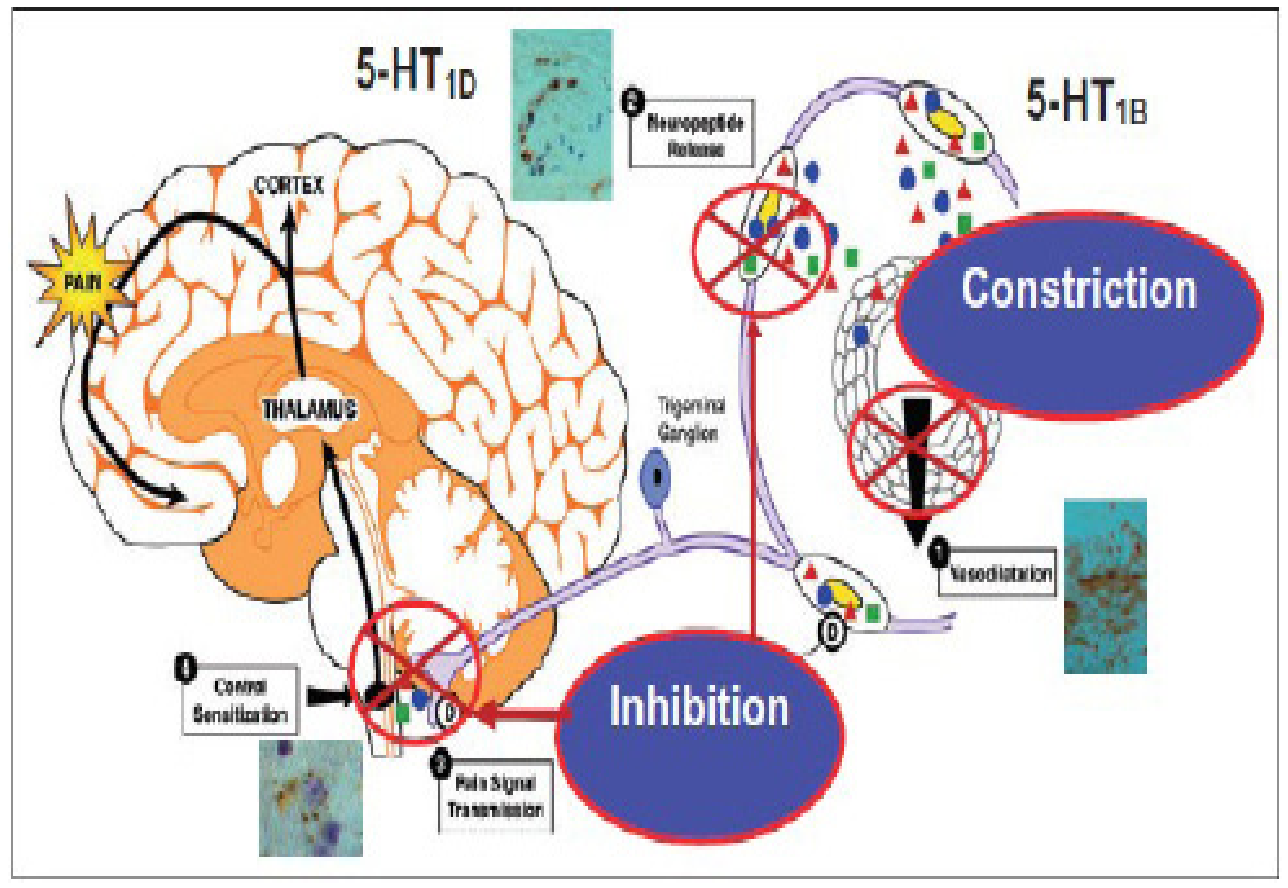

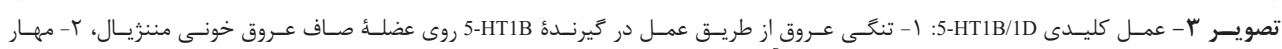

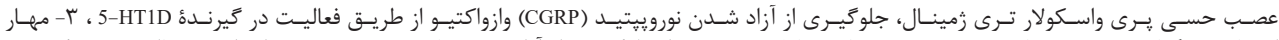

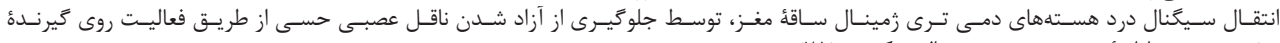

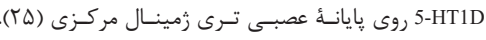




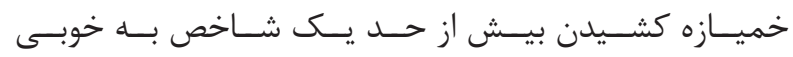

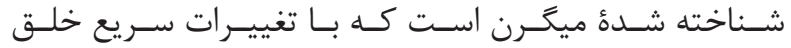

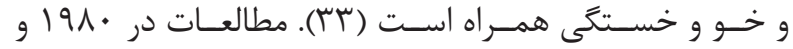

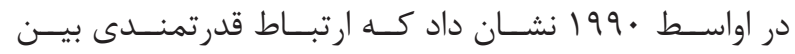

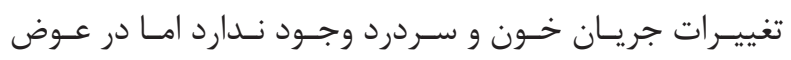

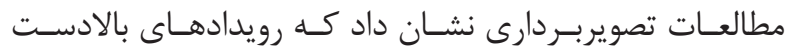

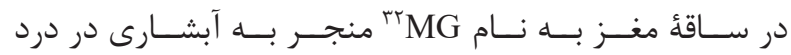

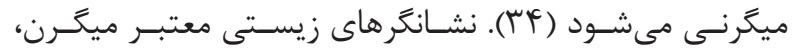

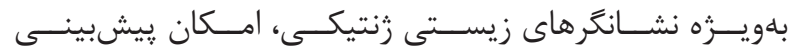

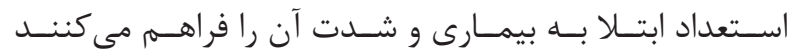

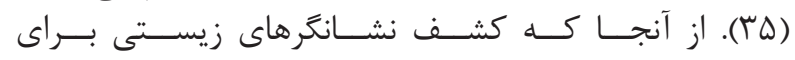

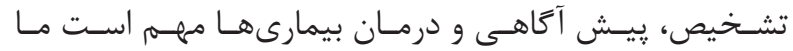

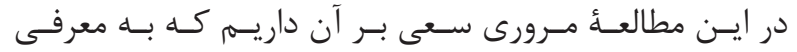

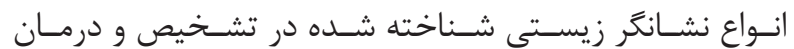

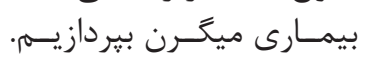

PubMed (http://www. مطالعـات از يايـعاه هــاى اطلاعاتـى ncbi.nih.gov/PubMed)، nature (https://www.nature. com)، springer (http://link.springer.com)، Google Scholar

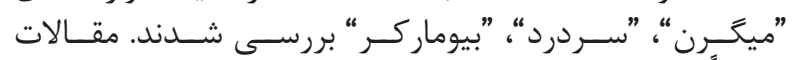

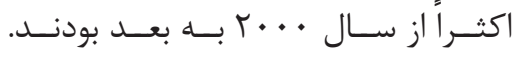

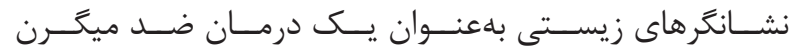

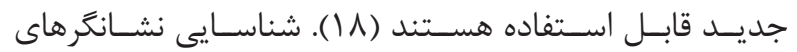

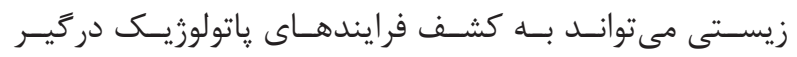

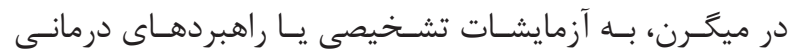

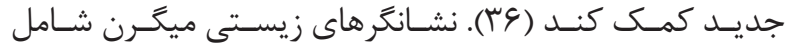

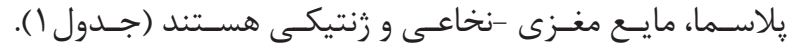

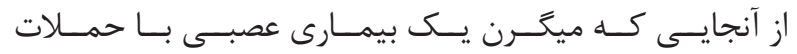

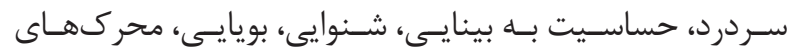

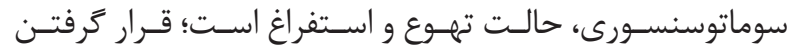

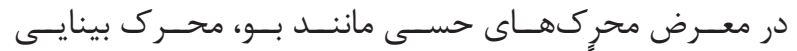

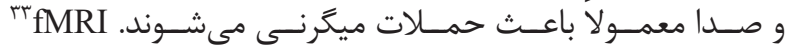

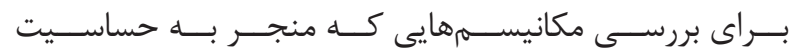

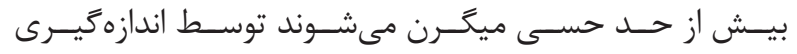

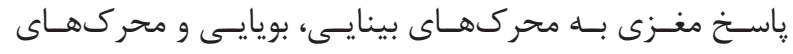

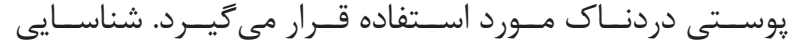

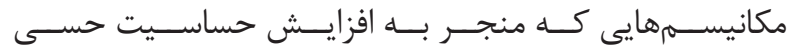

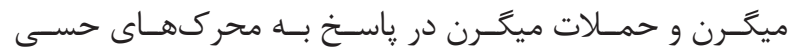

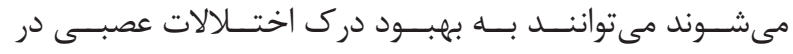

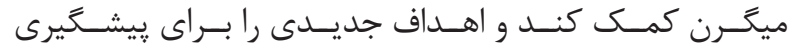

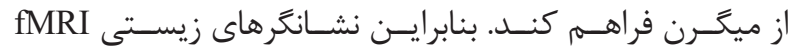

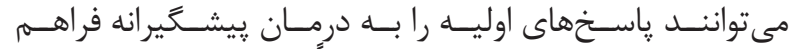

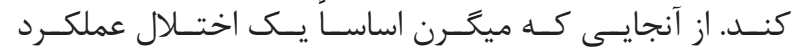

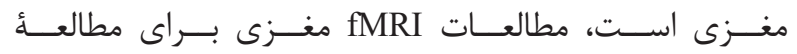

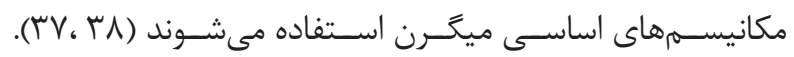

${ }^{30}$ Neuromodulation

${ }^{31}$ Biomarkers

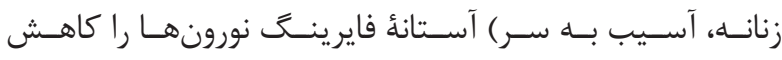

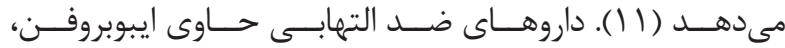

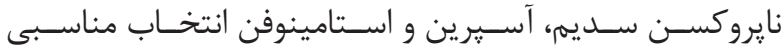

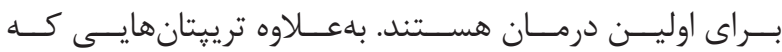

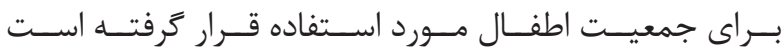

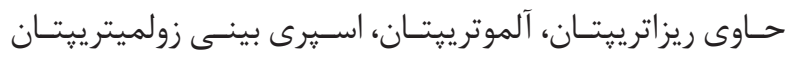

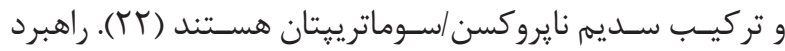

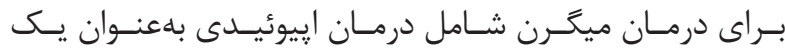

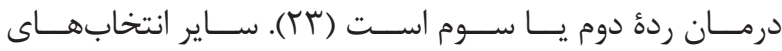

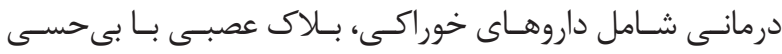

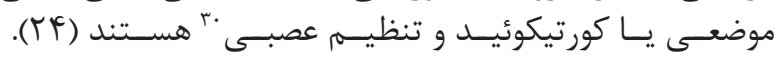

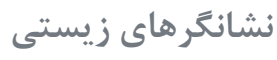

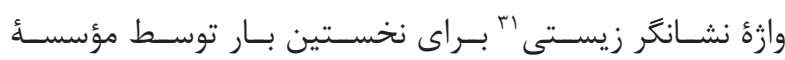

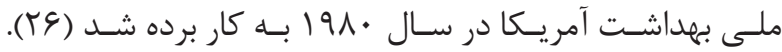

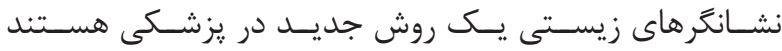

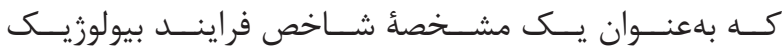

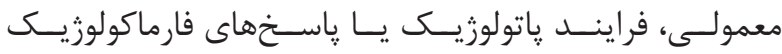

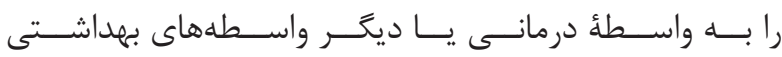

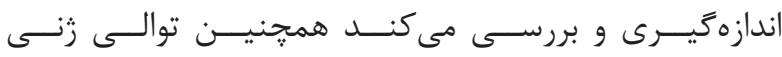
RNA/DNA

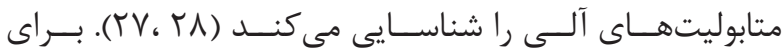

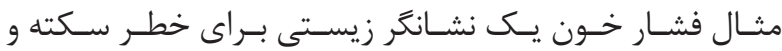

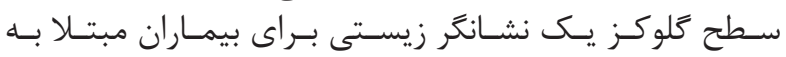

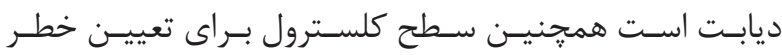

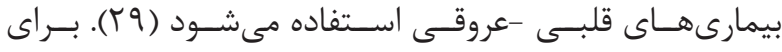

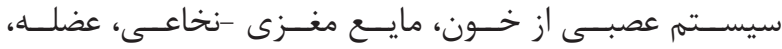

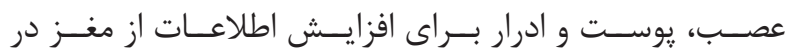

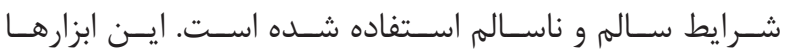

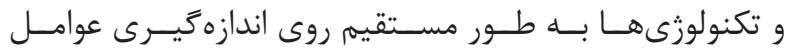

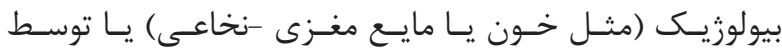

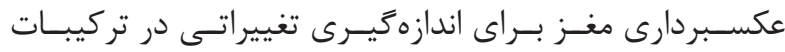

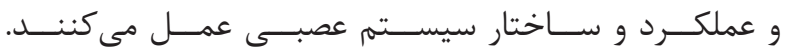

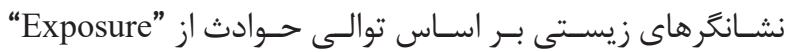

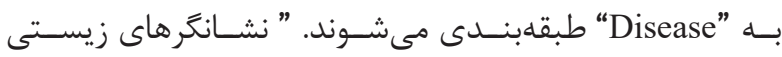
Exposure

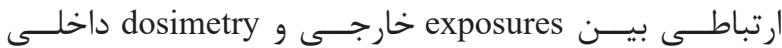

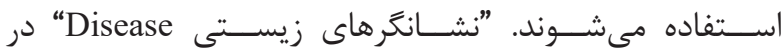

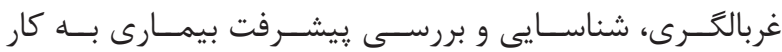

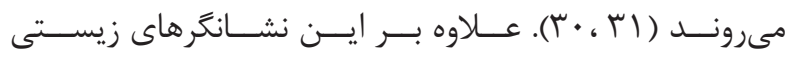

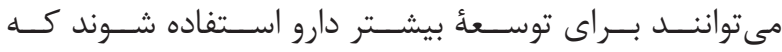

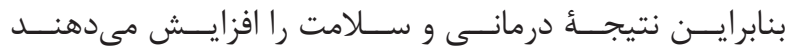

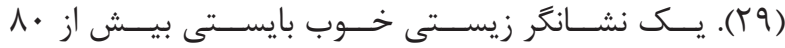

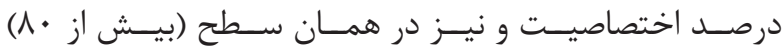

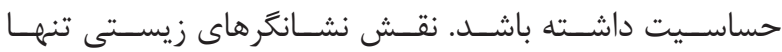

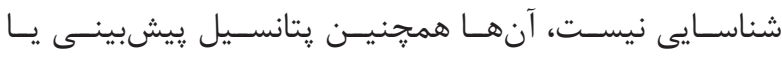

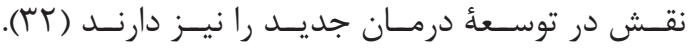

${ }^{32}$ Migraine generator

${ }^{33}$ Functional MRI 


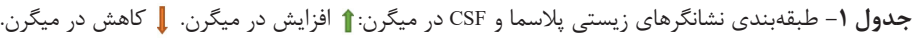

\begin{tabular}{|c|c|c|c|}
\hline \multicolumn{3}{|c|}{ نشانكر هاى زيستى } & ميكرن \\
\hline \multirow{17}{*}{ 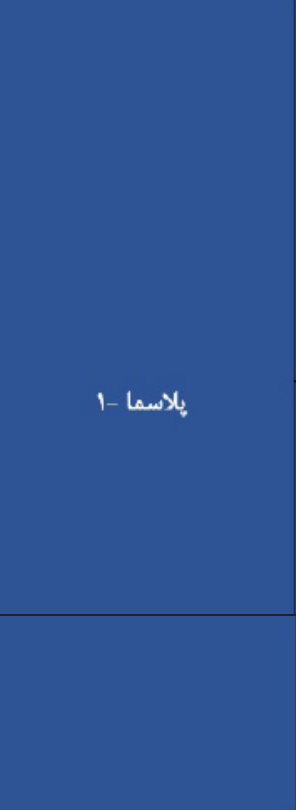 } & \multirow{8}{*}{ التهابى ا-1 } & 1-1-1 CRP & t \\
\hline & & $1-1-Y$ TNF- $\alpha$ & $\Uparrow$ \\
\hline & & $1-1-r \quad$ IL-6 & $\uparrow$ \\
\hline & & $1-1-F \quad I L-1 \beta$ & $\uparrow$ \\
\hline & & $1-1-\Delta \quad$ ILL-10 & $\downarrow$ \\
\hline & & $1-1-9$ TGF $-\beta$ & $\boldsymbol{1}$ \\
\hline & & 1-I-Y Adiponectin & $\downarrow$ \\
\hline & & $1-1-\Lambda$ Lipids & fexcept HDL \\
\hline & \multirow{5}{*}{ ترومبوز ولخته ץ-I } & $1-r-1$ Platelet & unknown \\
\hline & & $1-r-r \quad v W F$ & $\uparrow$ \\
\hline & & $1-r-r$ Fibrinogen & $\uparrow$ \\
\hline & & $1-T-F \quad$ PPA & $\downarrow$ \\
\hline & & $1-Y-\Delta \quad \mathbf{H C}$ & $\uparrow$ \\
\hline & \multirow{4}{*}{ فعاليت و اختلال عملكره ب-1 اندوتليال } & $1-r-1$ EMP & $\Uparrow$ \\
\hline & & $1-r-r$ ADMA & $\Uparrow$ \\
\hline & & $1-r-r$ Pro-BNP & $\Uparrow$ \\
\hline & & $1-r-F$ Oxidative stress & $\boldsymbol{\imath}$ \\
\hline \multirow{6}{*}{ r- مايع مغزى -زخاعى (CSF) } & \multicolumn{2}{|c|}{ Y-I Glutamate } & $\boldsymbol{\Uparrow}$ \\
\hline & $t-t$ & -EP & $\Downarrow$ \\
\hline & \multicolumn{2}{|c|}{ r-r CGRP } & $\uparrow$ \\
\hline & \multicolumn{2}{|c|}{ r-F NGF } & $\Uparrow$ \\
\hline & \multicolumn{2}{|c|}{$r-\Delta \quad$ HC } & $\Uparrow$ \\
\hline & \multicolumn{2}{|c|}{ T-9 GABA } & $\Uparrow$ \\
\hline
\end{tabular}

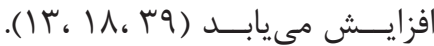

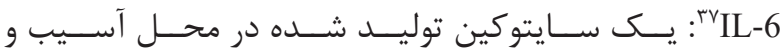

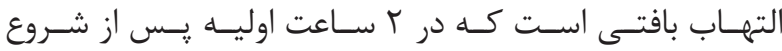

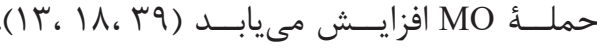

افز

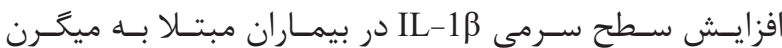

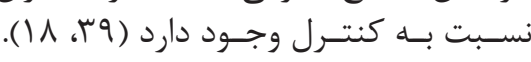

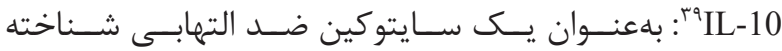

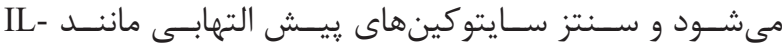

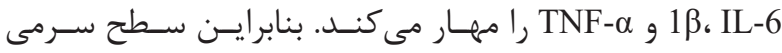

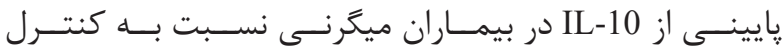

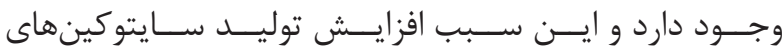

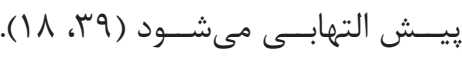

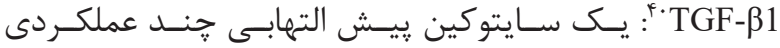

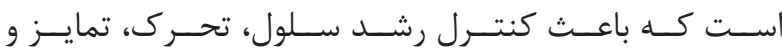

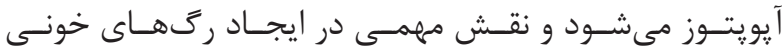

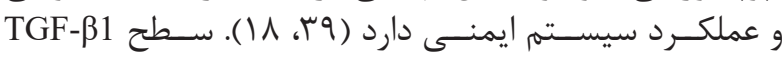

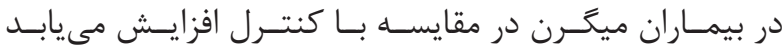

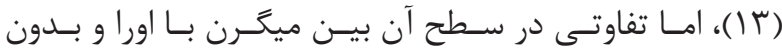

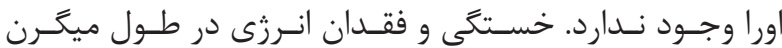

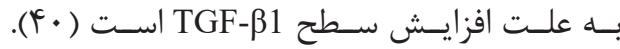

${ }^{34} \mathrm{C}$-reactive protein

${ }^{35}$ Fragment of crystallizable

${ }^{36}$ Tumor necrosis factor alpha

${ }^{37}$ Interleukin-6

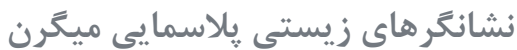

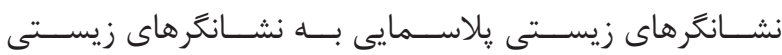

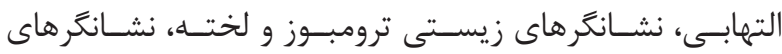

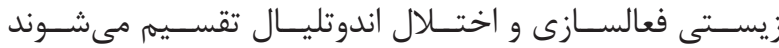

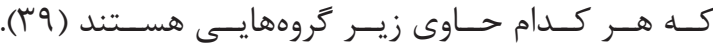
نشانكَر هاى زيستى التهابى و CRP

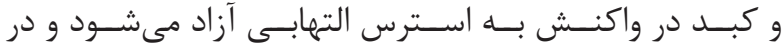

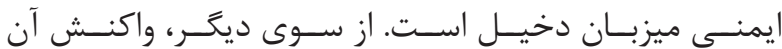

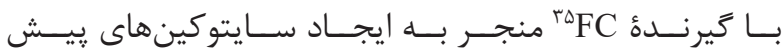

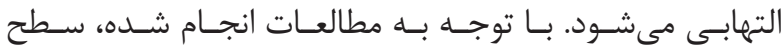

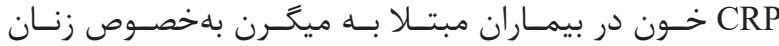

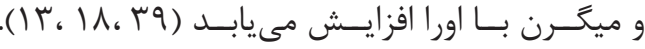

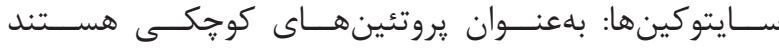

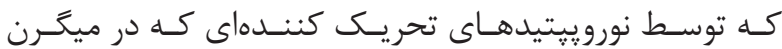

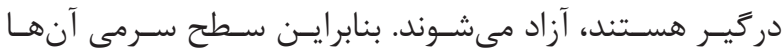

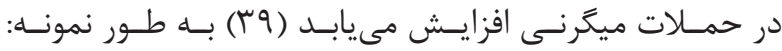
TNF- $\alpha$

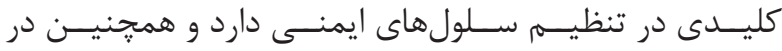

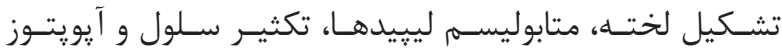

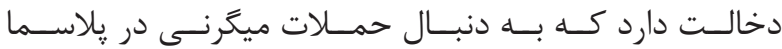

${ }^{38}$ Interleukin- $1 \beta$

${ }^{39}$ Interleukin-10

${ }^{40}$ Transforming growth factor beta 1 


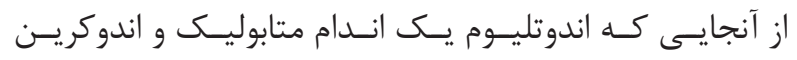

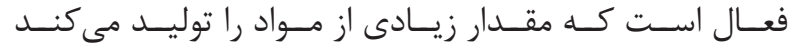

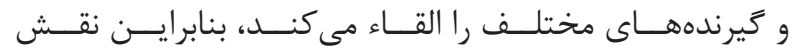

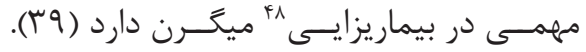
EMP

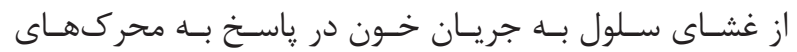

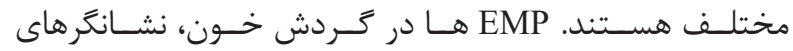

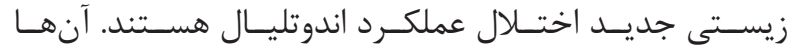

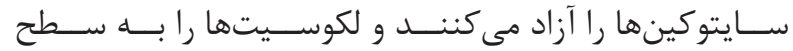

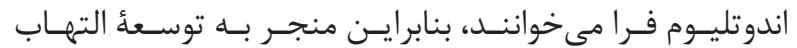

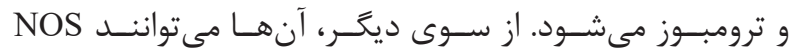

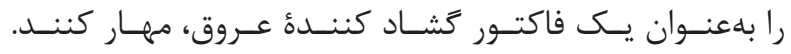

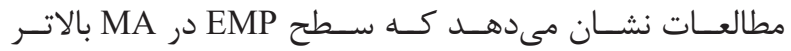

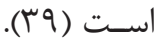

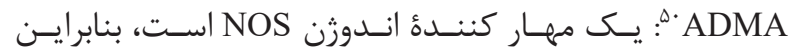

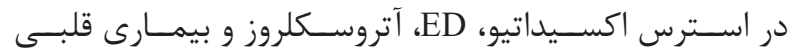

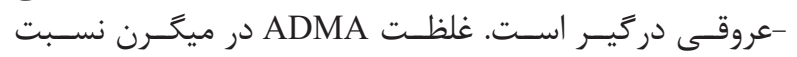

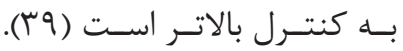

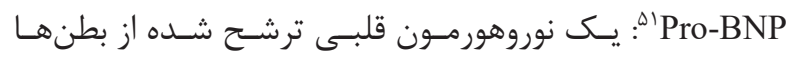

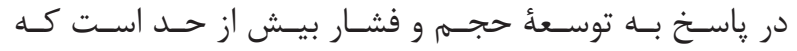

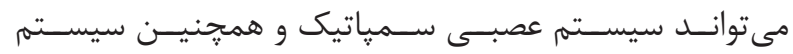

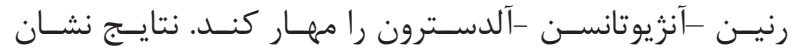

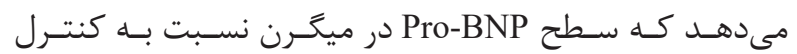

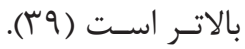

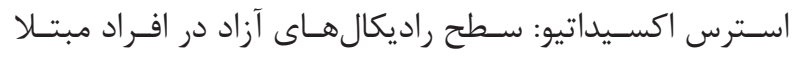

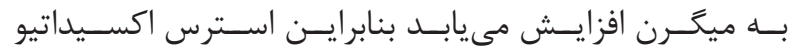

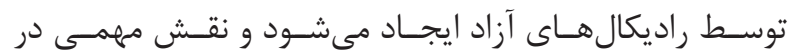

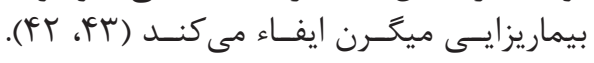

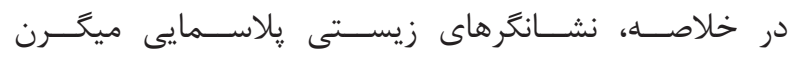

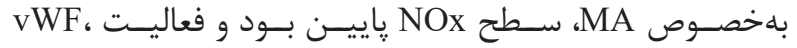

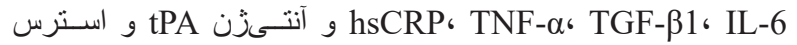

اكســيداتيو بـالا اســت (r) (I).

نشانَر هاى زيستى مايع مغزى -نخاعى ميگرن بrCSF

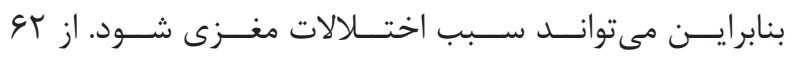

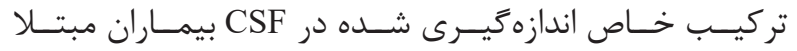

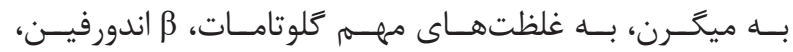

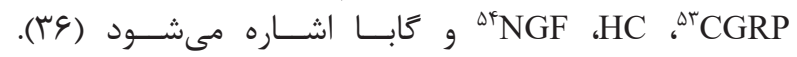

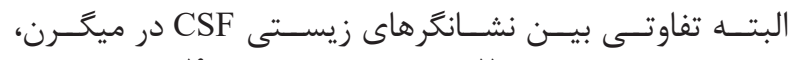

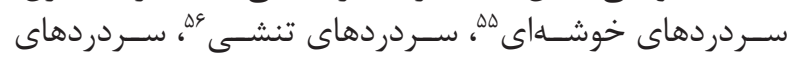

${ }^{41}$ Low-density lipoprotein cholesterol

${ }^{42}$ High-density lipoprotein cholesterol

${ }^{43}$ Vasoactivity

${ }^{44}$ Shear stress

${ }^{45}$ Von willebrand factor

${ }^{46}$ Tissue plasminogen activator

${ }^{47}$ Homocysteine

${ }^{48}$ Pathogenicity

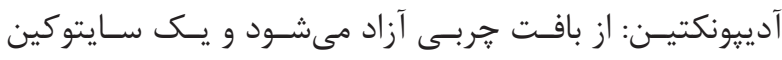

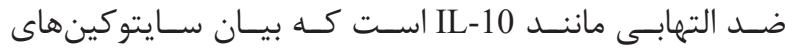

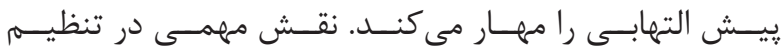

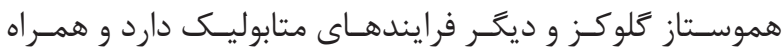

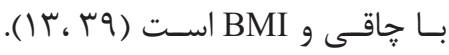

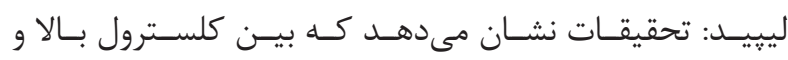

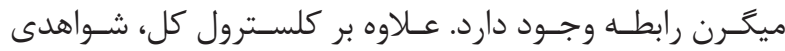

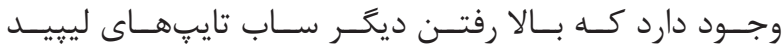

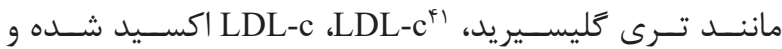

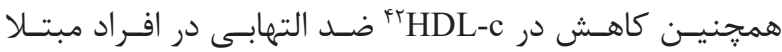

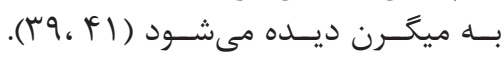
نشانَر هاى زيستى ترومبوز و لخته

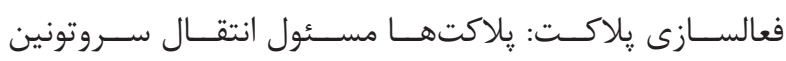

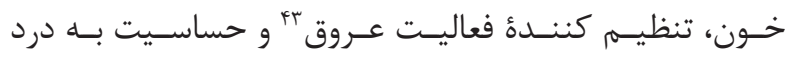

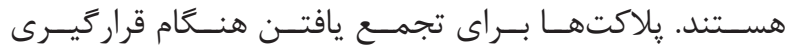

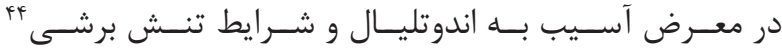

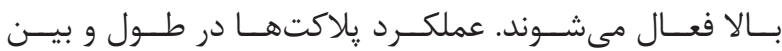

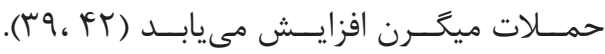

هـ

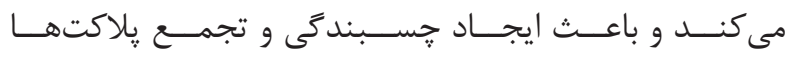

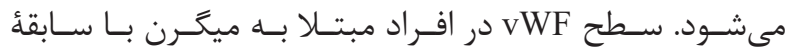

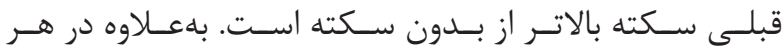

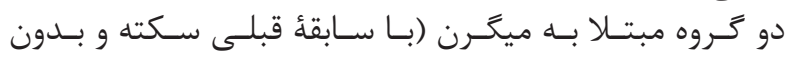

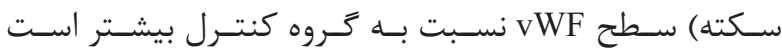

( (1)، I 1 ، rq, Fr)

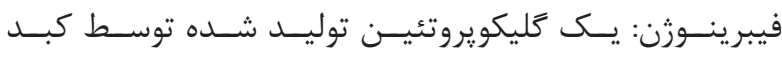

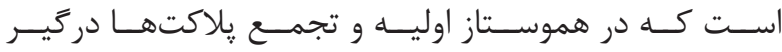

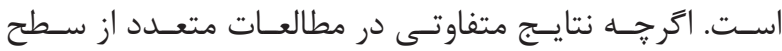

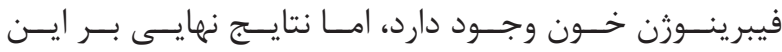

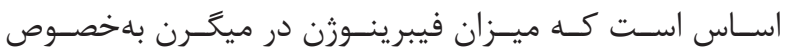

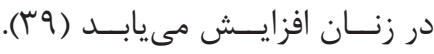
اخته هو

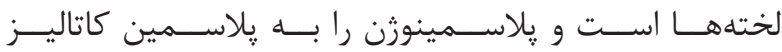

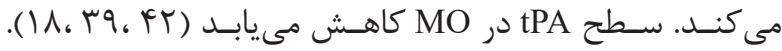

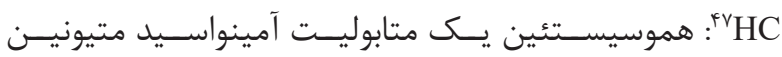

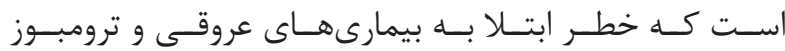

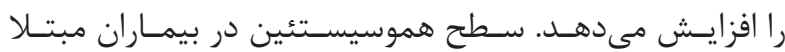

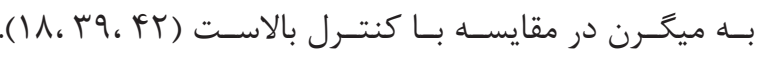

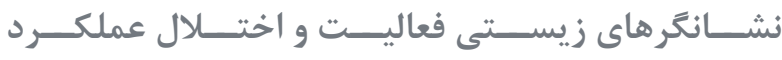
اندوتليــــال

\footnotetext{
${ }^{49}$ Endothelial microparticle

${ }^{50}$ Asymmetric dimethylarginine

${ }^{51}$ Pro-brain natruretic peptide

${ }^{52}$ Cerebrospinal fluid

${ }^{53}$ Calcitonin gene-related peptide

${ }^{54}$ Nerve growth factor

${ }^{55}$ Cluster headache

${ }^{56}$ Tension-type headeache
} 


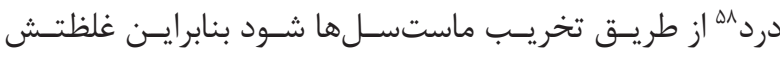

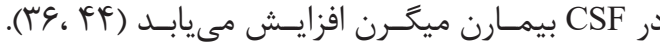

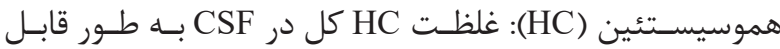

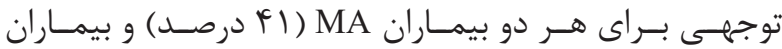

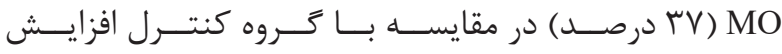

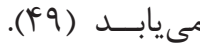

نشانغر هاى زيستى زُنتيكى ميكَرن

نشـانكَرهاى زيسـتى زنتيكسى، تغييـرات زنتيكسى (جهـش يـا

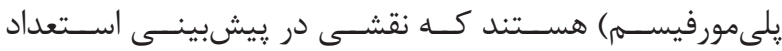

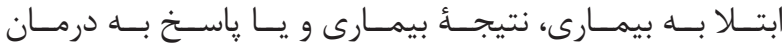

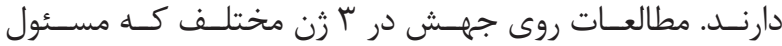

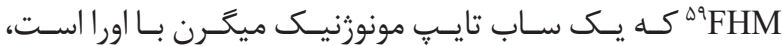

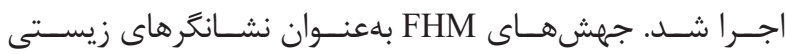

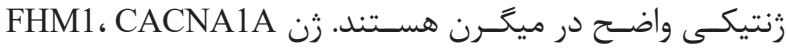

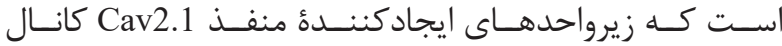

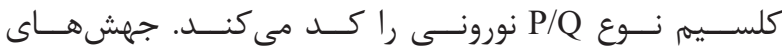

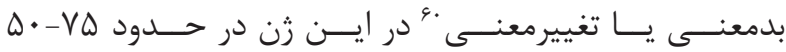

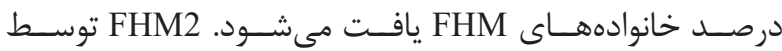

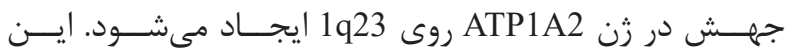

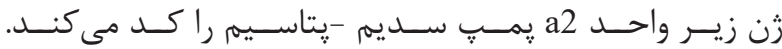

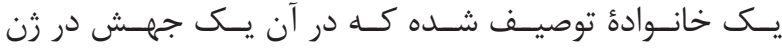

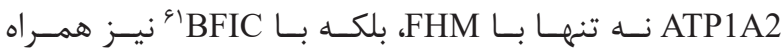

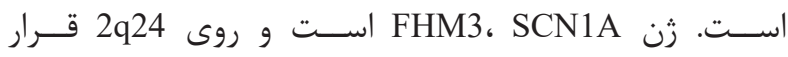

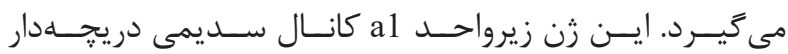

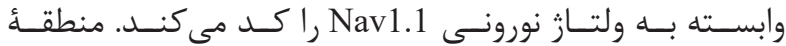

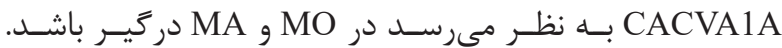

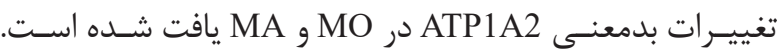

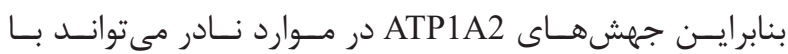

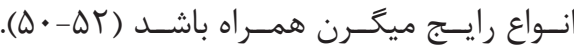

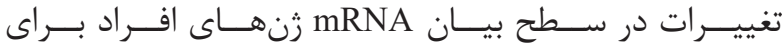

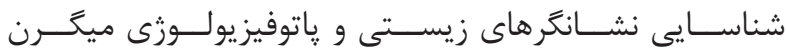

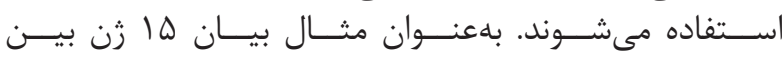

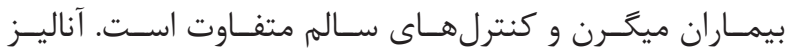

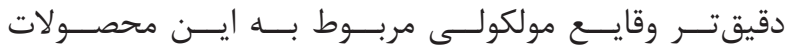

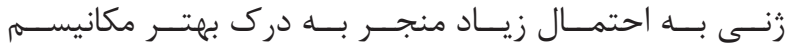

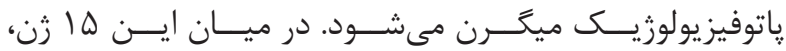
a-fodrin

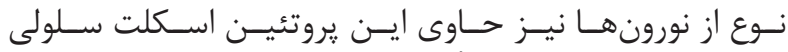

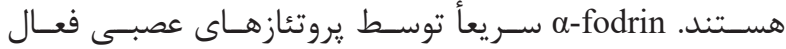

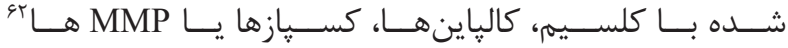

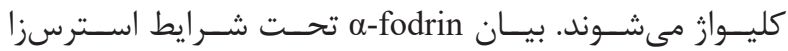

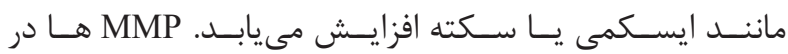

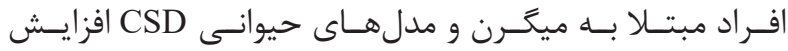

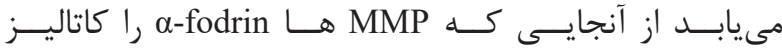

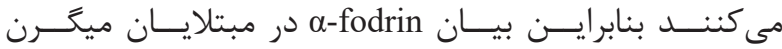

${ }^{57}$ Chronic tension-type headeache

${ }^{58}$ Hyperalgesia

${ }^{59}$ Familial hemiplegic migraine

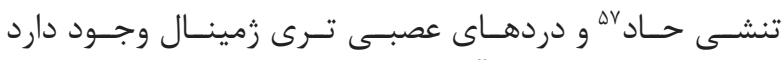

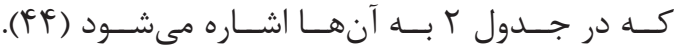

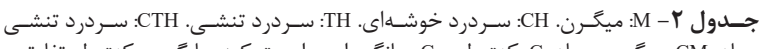

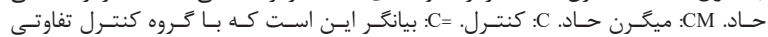

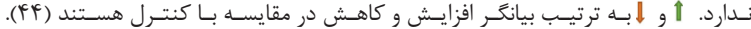

\begin{tabular}{|c|c|c|c|c|c|}
\hline & $\mathrm{M}$ & $\mathrm{CH}$ & $\mathrm{TH}$ & $\mathrm{CTH}$ & $\mathbf{C M}$ \\
\hline Glutamate & $\Uparrow$ & & & & \\
\hline $\boldsymbol{\beta}$-EP & $\Downarrow$ & $=\mathrm{C}$ & & $=\mathrm{C}$ & $\Downarrow$ \\
\hline CGRP & $\Uparrow$ & & & & \\
\hline NGF & $\Uparrow$ & & & & \\
\hline GABA & $\Uparrow$ & & $=\mathrm{C}$ & & \\
\hline
\end{tabular}

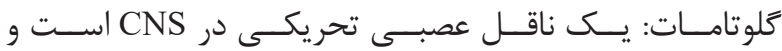

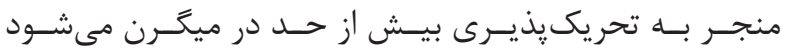

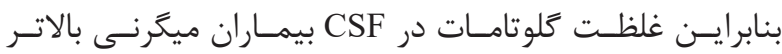

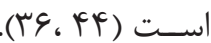

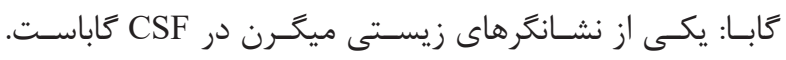

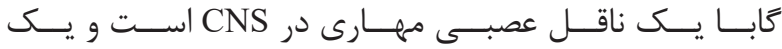

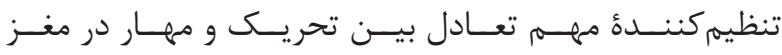

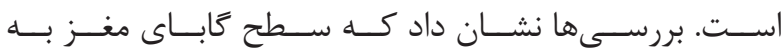

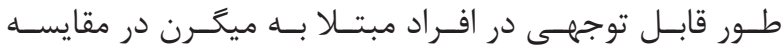

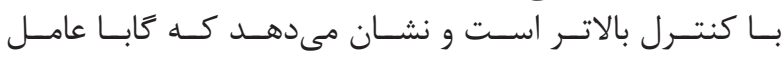

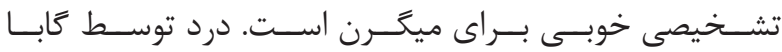

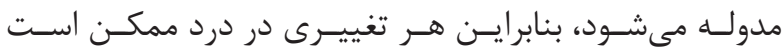

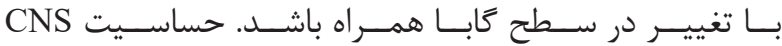

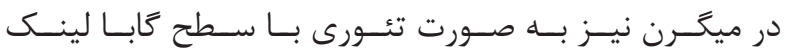

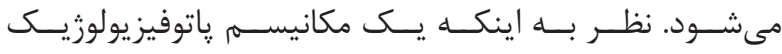

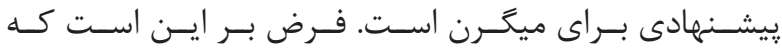

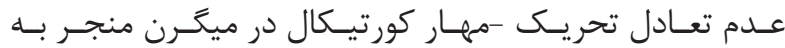

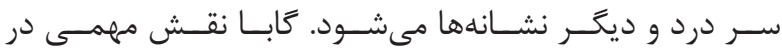

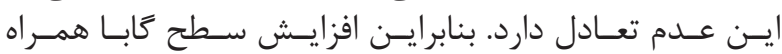

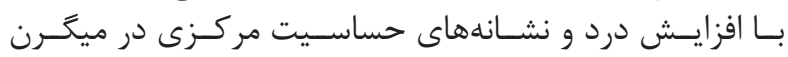

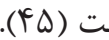

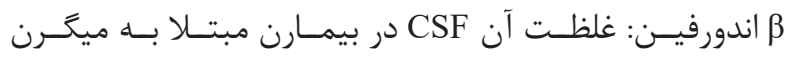

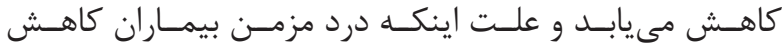

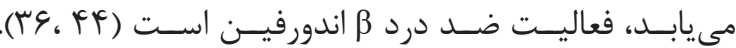

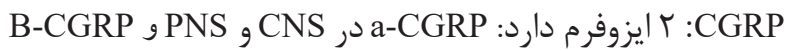

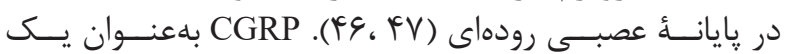

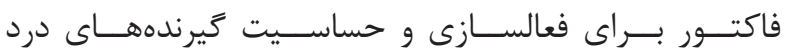

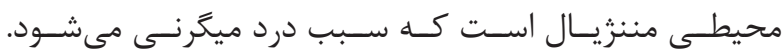

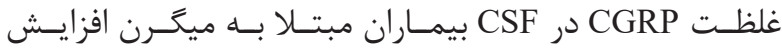

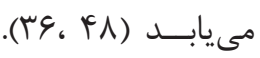

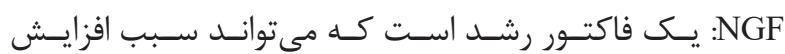
${ }^{60}$ Missense mutations
${ }^{61}$ Benign familial infantile convulsions
${ }^{62}$ Matrix metalloproteinases 


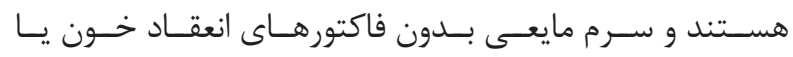

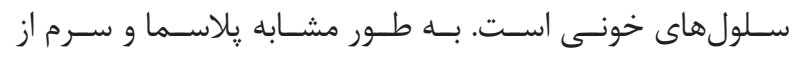

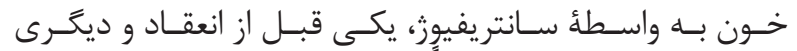

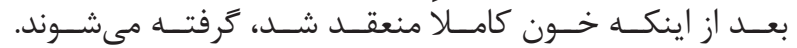

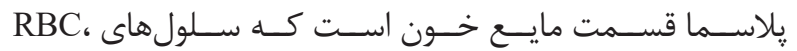

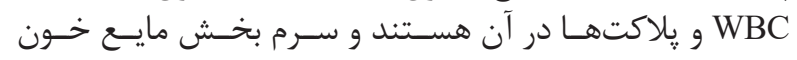

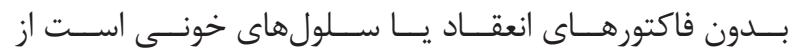

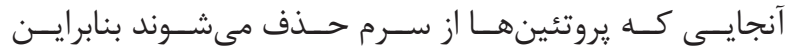

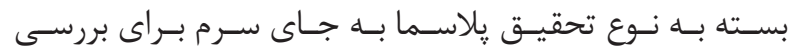

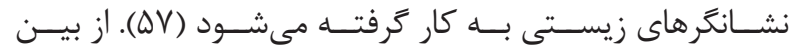

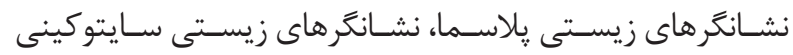

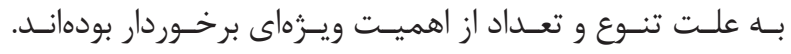

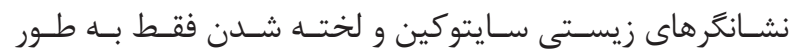

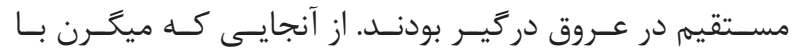

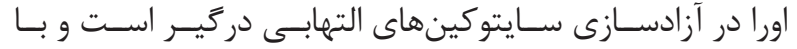

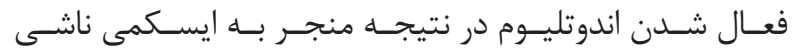

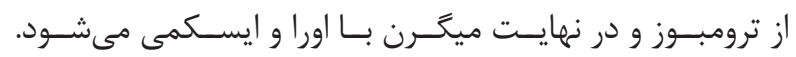

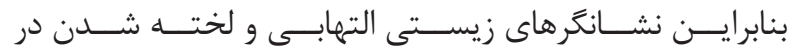

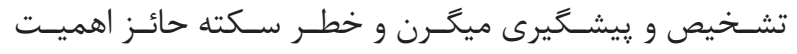

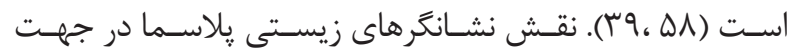

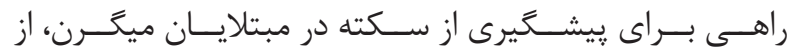

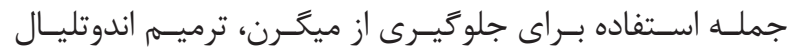

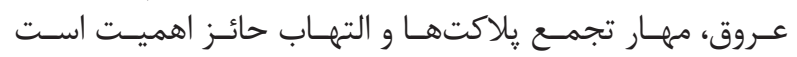

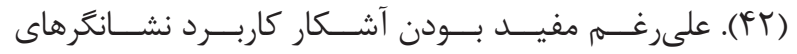

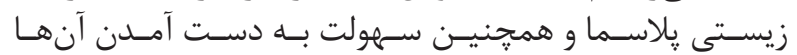

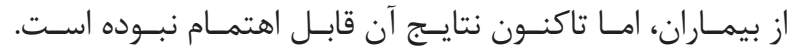

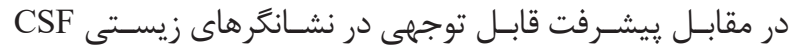

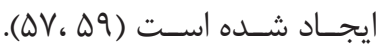

قايسـع اطــراف CNS CSF

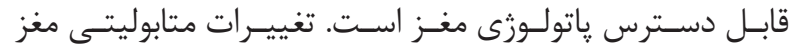

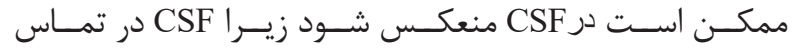

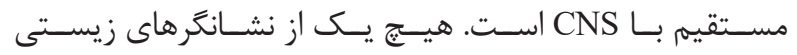

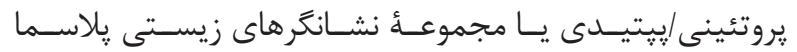

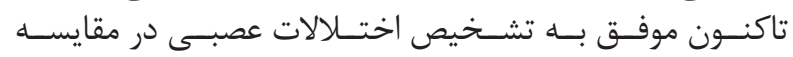

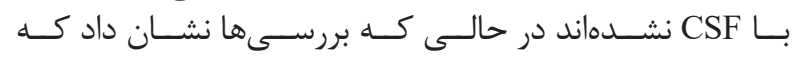

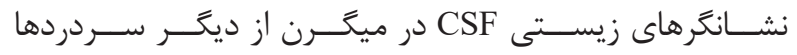

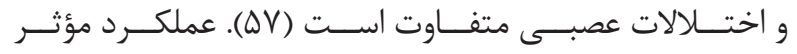

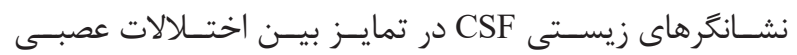

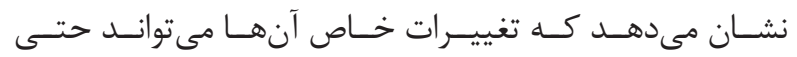

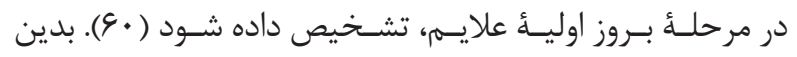

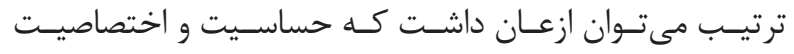

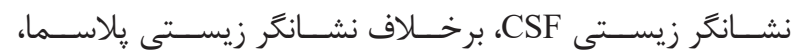

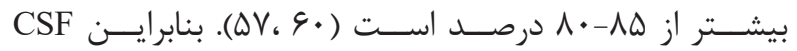

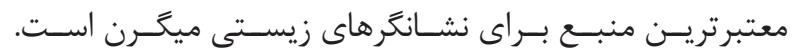

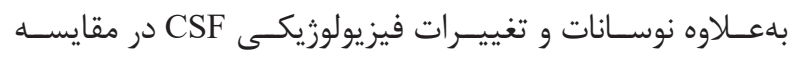

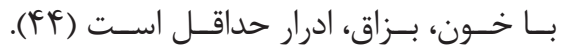

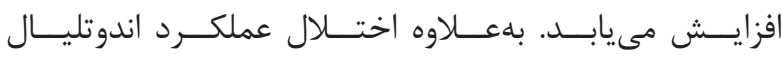

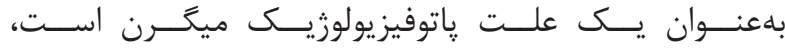

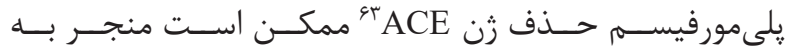

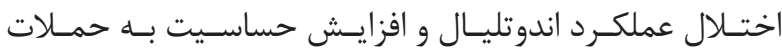

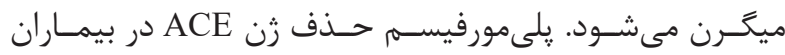

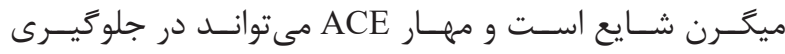

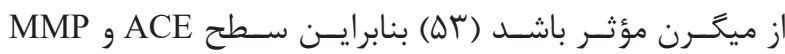

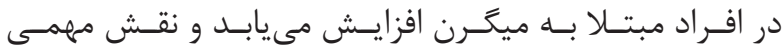

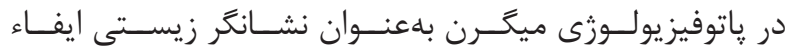

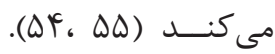

$$
\text { نشانًَر هاى زيستى درمانى }
$$

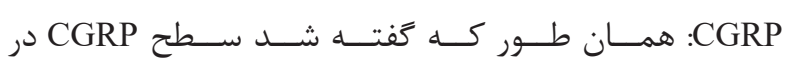

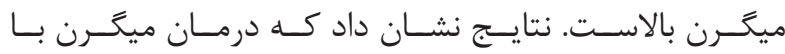

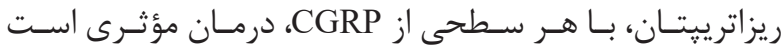

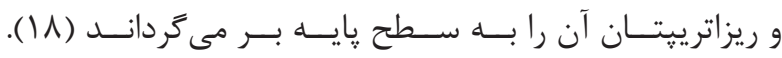

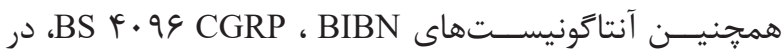

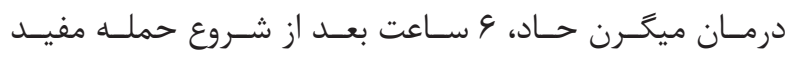

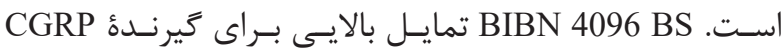
انســان دارد (ه) (ه).

PG

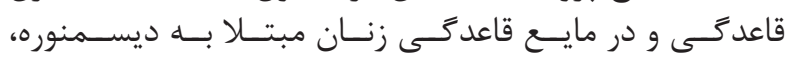

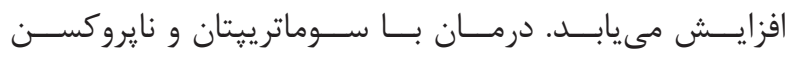

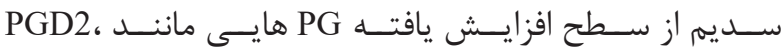

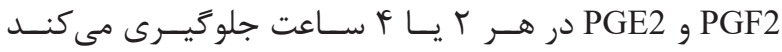

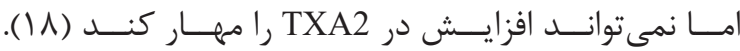

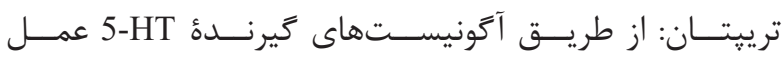

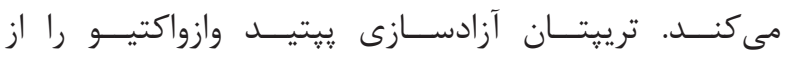

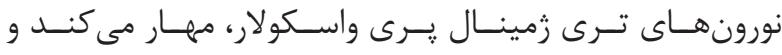

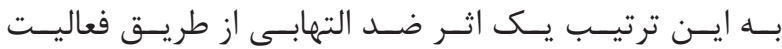

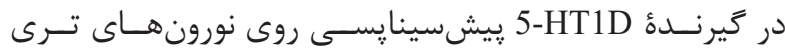

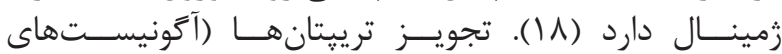

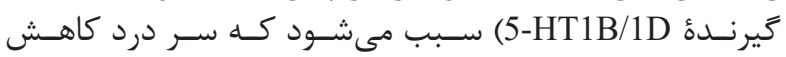

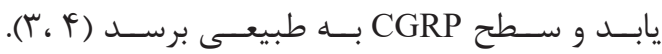
فيرهاى

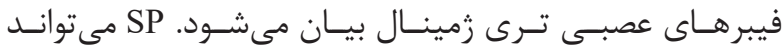

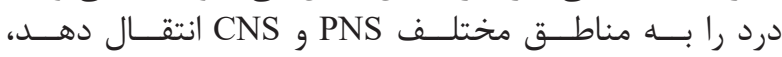

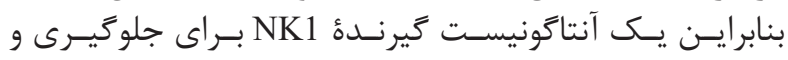

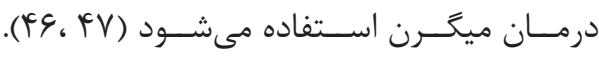
نتيجه

تعـداد و تنــوع نشـانگر هاى زيسـتى زنتيكـى در ميخـــرن بسـيار

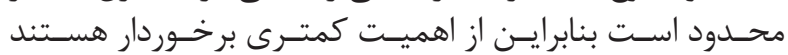

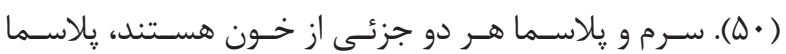

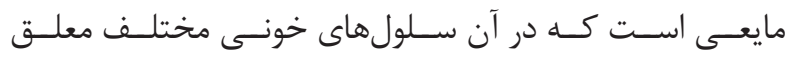

\footnotetext{
${ }^{63}$ Angiotensin-converting enzyme

${ }^{64}$ Prostaglandins

${ }^{65}$ Substance P
} 
1. Beres SJ, Liu GT. New advancements in migraine assessment and treatment. Advances in Ophthalmology and Optometry. 2016; 1(1): 249-60.

2. Goadsby P, Schoenen J, Ferrari M, Silberstein S, Dodick D. Towards a definition of intractable headache for use in clinical practice and trials. Cephalalgia. 2006; 26(9): 1168-70.

3. Edvinsson L, Villalón CM, MaassenVan Den Brink A. Basic mechanisms of migraine and its acute treatment. Pharmacol Ther. 2012; 136(3): 319-33.

4. Pascual J. CGRP antibodies: the holy grail for migraine prevention? Lancet Neurol. 2015; 14(11): 1066-7.

5. EvANs RW. The clinical features of migraine with and without aura. Pract Neurol. 2014; 13: 26-32.

6. Olesen J, Ashina M. Emerging migraine treatments and drug targets. Trends Pharmacol Sci. 2011; 32(6): 352-9.

7. Zagami AS. Pathophysiology of migraine and tensiontype headache. Curr Opin Neurol. 1994; 7(3): 272-7.

8. Wessman M, Terwindt GM, Kaunisto MA, Palotie A, Ophoff RA. Migraine: a complex genetic disorder. Lancet Neurol. 2007; 6(6): 521-32.

9. Ligthart L, Boomsma DI, Martin NG, Stubbe JH, Nyholt DR. Migraine with aura and migraine without aura are not distinct entities: further evidence from a large dutch population study. Twin Res Hum Genet. 2006; 9(1): 54-63.

10. Finkel AG, Yerry JA, Mann JD. Dietary considerations in migraine management: does a consistent diet improve migraine? Curr Pain Headache Rep. 2013; 17(11): 373. doi: 10.1007/s11916-013-0373-4.

11. Ramadan NM, Buchanan TM. New and future migraine therapy. Pharmacol Ther. 2006; 112(1): 199212.

12. Vetvik KG, MacGregor EA. Sex differences in the epidemiology, clinical features, and pathophysiology of migraine. Lancet Neurol. 2017; 16(1): 76-87.

13. Tietjen GE, Khubchandani J, Herial NA, Shah K. Adverse childhood experiences are associated with migraine and vascular biomarkers. Headache. 2012; 52(6): 920-9.

14. Van der Schueren BJ, Verbrugge FH, Verbesselt R, Hecken AV, Depré M, de Hoon JN. No arguments for increased endothelial nitric oxide synthase activity in migraine based on peripheral biomarkers. Cephalalgia. 2010; 30(11): 1354-65.

15. Kokavec A. Migraine: A disorder of metabolism? Med Hypotheses. 2016; 97: 117-30.

16. Salmito MC, Duarte JA, Morganti LOG, Brandão PVC, Nakao BH, Villa TR, et al. Prophylactic treatment of vestibular migraine. Braz J Otorhinolaryngol. 2017; 83(4): 404-10.

17. Benoit DP. An introduction to migraine pathophysiology. Tech Reg Anesth Pain Manag. 2009; 13(1): 5-8.

18. Durham P, Papapetropoulos S. Biomarkers associated with migraine and their potential role in migraine management. Headache. 2013; 53(8): 126277.

19. Just S, Arndt K, Weiser T, Doods H. Pathophysiology of migraine: a role for neuropeptides. Drug discovery today: Disease Mechanisms. 2006; 3(3): 327-33.

20. Zargaran A, Borhani-Haghighi A, Faridi P, Daneshamouz S, Mohagheghzadeh A. A review on the management of migraine in the avicenna's canon of medicine. Neurol Sci. 2016; 37(3): 471-8.

21. Hargreaves R, Shepheard S. Pathophysiology of migraine-new insights. Can J Neurol Sci. 1999; 26(3): 12-9.

22. Kacperski J, Hershey AD . Newly approved agents for the treatment and prevention of pediatric migraine. CNS Drugs. 2016; 30(9): 837-44.

23. Stone MT, Weed V, Kulich RJ. Opioid treatment of migraine: Risk factors and behavioral issues. Curr Pain Headache Rep. 2016; 20(9): 51. doi: 10.1007/s11916016-0581-9.

24. May A, Schulte LH. Chronic migraine: risk factors, mechanisms and treatment. Nat Rev Neurol. 2016; 12(8): $455-64$.

25. Hargreaves RJ, Lines CR, Rapoport AM, Ho TW, Sheftell FD. Ten years of rizatriptan: from development to clinical science and future directions. Headache. 2009; 49: S3-S20.

26. Aronson JK. Biomarkers and surrogate endpoints. Br J Clin Pharmacol. 2005; 59(5): 491-4.

27. Vaughan L. Biomarkers in acute medicine. Medicine. 2013; 41(3): 136-41. 
28. Tanaka T, Tanaka M, Tanaka T, Ishigamori R. Biomarkers for colorectal cancer. Int J Mol Sci. 2010; 11(9): 3209-25.

29. McCormick T, Martin K, Hehenberger M. The evolving role of biomarkers: Focusing on patients from research to clinical practice. IBM Global Business Services. 2007.

30. Mayeux R. Biomarkers: potential uses and limitations. Neuro Rx. 2004; 1(2): 182-8.

31. Gârban Z, Avacovici A, Gârban G, Ghibu G, Velciov AB, Pop CI. Biomarkers: theoretical aspects and applicative peculiarities note i. general characteristics of biomarkers. Agroalimentary Process Technol. 2005; 11: 139-46.

32. Polivka J, Krakorova K, Peterka M, Topolcan O. Current status of biomarker research in neurology. EPMA Journal. 2016; 7(1): 14. doi: 10.1186/s13167016-0063-5.

33. Dahlem MA, Kurths J, Ferrari MD, Aihara K, Scheffer M, May A. Understanding migraine using dynamic network biomarkers. Cephalalgia. 2015; 35(7): 627-30.

34. Dahlem M, Rode S, May A, Fujiwara N, Hirata Y, Aihara K, et al. Towards dynamical network biomarkers in neuromodulation of episodic migraine. Translational Neuroscience. 2013; 4(3): 282-94.

35. Kondratieva N, Azimova J, Skorobogatykh K, Sergeev A, Naumova E, Kokaeva Z, et al. Biomarkers of migraine: Part 1-Genetic markers. J Neurol Sci. 2016; 369: 63-76.

36. van Dongen RM, Zielman R, Noga M, Dekkers OM, Hankemeier T, van den Maagdenberg AM, et al. Migraine biomarkers in cerebrospinal fluid: a systematic review and meta-analysis. Cephalalgia. 2017; 37(1): 49-63.

37. Schwedt TJ, Chiang C-C, Chong CD, Dodick DW. Functional MRI of migraine. The Lancet Neurology. 2015; 14(1): 81-91.

38. Wang M, Su J, Zhang J, Zhao Y, Yao Q, Zhang Q, et al. Visual cortex and cerebellum hyperactivation during negative emotion picture stimuli in migraine patients. Sci Rep. 2017; 7: 41919. doi: 10.1038/srep41919.

39. Tietjen GE, Khubchandani J. Vascular biomarkers in migraine. Cephalalgia. 2015; 35(2): 95-117.

40. Ishizaki K, Takeshima T, Fukuhara Y, Araki $\mathrm{H}$, Nakaso K, Kusumi M, et al. Increased plasma transforming growth factor- $\beta 1$ in migraine. Headache.
2005; 45(9): 1224-8.

41. Kurth T, Ridker P, Buring J. Migraine and biomarkers of cardiovascular disease in women. Cephalalgia. 2008; 28(1): 49-56.

42. Tietjen GE, Herial NA, White L, Utley C, Kosmyna JM, Khuder SA. Migraine and biomarkers of endothelial activation in young women. Stroke. 2009; 40(9): $2977-$ 82.

43. Yilmaz N, Aydin O, Yegin A, Tiltak A, Eren E. Increased levels of total oxidant status and decreased activity of arylesterase in migraineurs. Clin Biochem. 2011; 44(10-11): 832-7.

44. Harrington MG. Cerebrospinal fluid biomarkers in primary headache disorders. Headache. 2006; 46(7): 1075-87.

45. Aguila M-ER, Rebbeck T, Leaver AM, Lagopoulos J, Brennan PC, Hübscher M, et al. The association between clinical characteristics of migraine and brain GABA levels: an exploratory study. J Pain. 2016; 17(10): 1058-67.

46. Tajti J, Szok D, Majláth Z, Tuka B, Csáti A, Vécsei L. Migraine and neuropeptides. Neuropeptides. 2015; 52: $19-30$.

47. Matesanz F, Fedetz M, Barrionuevo C, Karaky M, Catalá-Rabasa A, Potenciano V, et al. A splice variant in the ACSL5 gene relates migraine with fatty acid activation in mitochondria. E Eur J Hum Genet. 2016; 24(11): 1572-7.

48. Cernuda-Morollón E, Larrosa D, Ramón C, Vega J, Martínez-Camblor P, Pascual J. Interictal increase of CGRP levels in peripheral blood as a biomarker for chronic migraine. Neurology. 2013; 81(14): 1191-6.

49. Isobe C, Terayama Y. A remarkable increase in total homocysteine concentrations in the CSF of migraine patients with aura. Headache. 2010; 50(10): 1561-9.

50. De Vries B, Haan J, Frants RR, Van den Maagdenberg AM, Ferrari MD. Genetic biomarkers for migraine. Headache. 2006; 46(7): 1059-68.

51. Fernandez F, Colson N, Griffiths LR. Pharmacogenetics of migraine: genetic variants and their potential role in migraine therapy. Pharmacogenomics. 2007; 8(6): 609-22.

52. De Vries B, Frants RR, Ferrari MD, van den Maagdenberg AM. Molecular genetics of migraine. Hum Genet. 2009; 126(1): 115-32.

53. Hershey AD. Genetics of migraine headache in 
children. Current Pain and Headache Reports. 2007; 11(5): 390-5.

54. Nagata E, Hattori $H$, Kato M, Ogasawara S, Suzuki S, Shibata M, et al. Identification of biomarkers associated with migraine with aura. Neurosci Res. 2009; 64(1): 104-10.

55. Imamura K, Takeshima T, Fusayasu E, Nakashima $\mathrm{K}$. Increased plasma matrix metalloproteinase-9 levels in migraineurs. Headache. 2008; 48(1): 135-9.

56. Olesen J, Diener H-C, Husstedt IW, Goadsby PJ, Hall D, Meier U, et al. Calcitonin gene-related peptide receptor antagonist BIBN $4096 \mathrm{BS}$ for the acute treatment of migraine. N Engl J Med. 2004; 350(11): 1104-10.

57. Aluise CD, Sowell RA, Butterfield DA. Peptides and proteins in plasma and cerebrospinal fluid as biomarkers for the prediction, diagnosis, and monitoring of therapeutic efficacy of Alzheimer's disease. Biochim Biophys Acta. 2008; 1782(10): 549-58.

58. Ceylan M, Bayraktutan OF, Becel S, Atis Ö, Yalcin A, Kotan D. Serum levels of pentraxin-3 and other inflammatory biomarkers in migraine: association with migraine characteristics. Cephalalgia. 2016; 36(6): 518-25.

59. Gonzalez-Cuyar LF, Sonnen JA, Montine KS, Keene CD, Montine TJ. Role of cerebrospinal fluid and plasma biomarkers in the diagnosis of neurodegenerative disorders and mild cognitive impairment. Curr Neurol Neurosci Rep. 2011; 11(5): 455-63.

60. Szalárdy L, Zádori D, Klivényi P, Vécsei L. The role of cerebrospinal fluid biomarkers in the evolution of diagnostic criteria in Alzheimer's disease: Shortcomings in prodromal diagnosis. J Alzheimers Dis. 2016; 53(2): 373-92. 